\title{
Multi-Disciplinary Optimization of Variable Rotor Speed and Active Blade Twist Rotorcraft: Trade-Off between Noise and Emissions
}

\author{
Nikolaos D. Polyzos ${ }^{\text {a,1 }}$, Stavros Vouros ${ }^{\text {a,* }}$, Ioannis Goulos a , Vassilios Pachidis ${ }^{\text {a }}$ \\ ${ }^{a}$ Centre for Propulsion Engineering, School of Aerospace, Transport and Manufacturing, Cranfield University, \\ Bedfordshire, MK430AL, UK. \\ ${ }^{1}$ Lead author. \\ * Corresponding author at: Centre for Propulsion Engineering, School of Aerospace, Transport and Manufacturing, \\ Cranfield University, Building 52, Room G54, Bedfordshire, MK430AL, UK, Tel.: +44 (0) 123475 8286, e-mail \\ address: vourosstavros@windowslive.com (S. Vouros).
}

\begin{abstract}
The concepts of variable rotor speed and active blade twist are emerging technologies for the next generation of civil rotorcraft. Previous research has focused on the optimum implementation of these technologies for improved fuel economy and environmental impact. Within this work, an integrated approach is deployed to quantify the concurrent reductions in rotor noise and $N O_{x}$ emissions. A relaxation-based free-wake inflow model, coupled with unsteady blade aerodynamics modeling, resolves the flow-field around the main rotor. Aero-acoustic predictions are performed through an acoustic-analogy-based formulation. Gaseous emissions are then predicted via stirred-reactor modeling, coupled with zero-dimensional engine performance analysis method. This strategy is incorporated into a multi-disciplinary genetic algorithm optimization process based on surrogate modeling. Optimal schedules of combined variable rotor speed and active blade twist controls are derived for a twin-engine light helicopter in descent. The accrued schedules suggest $N O_{x}$ reductions between $6 \%$ and $21 \%$, simultaneously with sourcenoise reductions of the order of $2-8 \mathrm{~dB}$, relative to the non-morphing rotor case. The developed strategy constitutes an enabling methodology for the holistic and multi-disciplinary assessment of morphing helicopter rotor configurations.
\end{abstract}


Keywords: Helicopters, Aerodynamics, Aeroacoustics, Gas turbines, Multi-disciplinary optimization, Environmental impact.

\section{Nomenclature}

\section{Roman Symbols}

$A U M$

$C_{L}$

$C_{M}$

$C_{T}$

EAOASPL

$\triangle E A O A S P L$

$h$

$L$

$M$

$\vec{M}$

MTOW

$N$

$N_{\text {Pearson }}$

$N O_{x}$

$N O_{x}^{\text {rate }}$
All-up mass

$[\mathrm{kg}]$

Rolling moment coefficient $L /\left(\rho \pi \Omega^{2} R^{5}\right)$

$[-]$

Pitching moment coefficient $M /\left(\rho \pi \Omega^{2} R^{5}\right)$

$[-]$

Thrust coefficient $T /\left(\rho \pi \Omega^{2} R^{4}\right)$

$[-]$

Energy averaged overall sound pressure level

[dB]

EAOASPL difference relative to nominal rotor control settings

Density altitude

$[\mathrm{m}]$

Rotor rolling moment

$[\mathrm{Nm}]$

Rotor pitching moment

$[\mathrm{Nm}]$

Relative Mach number vector

$[-]$

Maximum take-off weight

$[\mathrm{kg}]$

Number of data points across the surface of the acoustic sphere

$[-]$

Pearson's product moment of correlation

$[-]$

Nitrogen oxide emissions

$[\mathrm{kg}]$

Rate of $N O_{x}$ formation

$[\mathrm{kg} / \mathrm{sec}]$ 


$\begin{array}{llc}\Lambda N O_{x}^{\text {rate }}[\%] & \begin{array}{l}\text { Change of } N O_{x}^{\text {rate }} \text { relative to nominal rotor control } \\ \text { settings }\end{array} & {[-]} \\ N R M S E & \text { Normalized mean root square error } & {[\%]} \\ \text { OASPL } & \text { Overall sound pressure level } & {[\mathrm{dB}]} \\ R & \text { Main rotor radius } & {[\mathrm{m}]} \\ \vec{r} & \text { Radiation distance vector } & {[\mathrm{m}]} \\ S F C & \text { Specific fuel consumption } & {[\mathrm{kg} / \mathrm{sec}]} \\ T & \text { Rotor thrust } & {[\mathrm{N}]} \\ V_{x} & \text { Flight speed } & {[\mathrm{m} / \mathrm{s}]} \\ V_{z} & \text { Climb rate } & {[\mathrm{m} / \mathrm{s}]}\end{array}$

\section{Greek Symbols}

$a_{s} \quad$ Wind-tunnel rotor shaft angle [deg]

$\theta_{t w} \quad$ Blade twist angle [deg]

$\theta_{1 S} \quad$ Longitudinal cyclic blade pitch $\quad[\mathrm{deg}]$

$\theta_{1 C} \quad$ Lateral cyclic blade pitch $\quad[\mathrm{deg}]$

$\rho \quad$ Air density $\quad\left[\mathrm{kg} / \mathrm{m}^{3}\right]$

$\sigma \quad$ Standard deviation $\quad[-]$

$\psi \quad$ Blade azimuthal position $\quad$ [deg]

$\Omega \quad$ Rotor speed $\quad[\mathrm{rad} / \mathrm{s}]$ 


\section{Superscripts}
()$^{O p t}$
Optimized quantity
()$^{\text {Nom }}$
Nominal quantity

\section{Introduction}

The environmental and acoustic impact of rotorcraft activities is expected to develop sharply in the near future due to the anticipated growth in civil operations [1]. To that end, advisory and regulatory bodies are adopting stricter goals and measures on pollutant emissions and noise, especially over or near populated areas. Landing and Take-Off (LTO) cycles constitute a priority for operators with respect to $N O_{x}$ emissions around airports [2]. During descending flight, increased noise emissions occur due to the intensified Blade Vortex Interaction (BVI) phenomena in the vicinity of the main rotor. The International Civil Aviation Organization (ICAO) proposed the so called "Balanced Approach" [3] for aircraft noise management in the vicinity of airports. This promotes the achievement of maximum acoustic benefits in the most cost-efficient way through combination of the following measures: (a) reduction of noise at source, (b) land-use planning and management, (c) noise abatement operational procedures, and (d) operational restrictions. An effective way of reducing aeroacoustic emissions is through mitigating adverse aerodynamic effects directly at source, meaning the vicinity of the main rotor. Due to the diverse nature of helicopter operational scenarios, active rotor control and morphing technologies have shown potential to improve operational performance, as well as reduce the environmental impact of rotorcraft.

Conventional helicopter rotors operate at near-constant rotational speeds, based on a trade-off between hover, maximum range speed and high-speed flight accounting also for resonant frequencies and rotor induced vibrations. However, it has been shown that there is potential to 
improve rotorcraft performance by appropriately varying the rotor-speed throughout the aircraft operational envelope [4]; [5]. Steiner [6] investigated the performance benefits and trim requirements by implementing variable rotor speed (VRS) on a UH-60 helicopter, showing $12 \%$ and $17 \%$ reductions in power requirement at hover and cruise near $60 \mathrm{~m} / \mathrm{s}$, respectively. BowenDavies and Chopra [7] examined the impact of a slowed main rotor on the aeromechanics and performance of a UH-60A helicopter in steady level flight, using the University of Maryland Advanced Rotorcraft Code (UMARC) [8]. For low-thrust requirements at low-speed cruise, a reduction in power requirement of up to $20 \%$ was observed with a rotor speed close to $76 \%$ of its nominal value. Mistry and Gandhi [9] investigated the impact of VRS combined with variable rotor radius on the performance of a UH-60A helicopter. Power savings of up to $14 \%$ in low-andlight conditions were achieved by rotor speed reduction alone. Misté et al. [4] presented a numerical approach for coupled helicopter-engine performance to assess the benefits arising from the implementation of variable rotor speed on a UH-60 helicopter equipped with two GE T700 turboshaft engines. Fuel savings of approximately $7-8 \%$ were estimated within a $\pm 15 \%$ range of the nominal rotor speed. The impact of VRS on rotor noise generation has been recognized [10]. The use of slowed rotors mitigates source noise generation due to the reduced magnitude of blade relative Mach number vector in the direction of noise radiation. However, the concept of VRS has never been systematically investigated towards the trade-off between $N O_{x}$ and noise impact. Furthermore, there has not been a comprehensive analysis to highlight the impact of optimum scheduling.

Active rotor morphing [11] aims to abate adverse effects associated with rotor aeromechanics by inducing time-dependent variations of blade geometry. Variable blade pitch [12] can be implemented either through individual blade control (IBC) [13] or in a collective manner, through higher harmonic control (HHC) inputs [14] over the collective and cyclic pitch or by active blade twist (ABT) variations [15]. BVIs are of the most annoying noise sources during rotorcraft operation, typically occurring in descending flight where blade tip vortices are in close proximity 
to the elastic rotor blades. Brooks and Booth [16] examined the effects of HHC on BVI noise and vibrations, using a dynamically scaled, four-bladed, articulated rotor in low-speed descent conditions. It was observed that 4/rev HHC excitations achieved BVI noise reductions of up to $5.6 \mathrm{~dB}$; however, low-frequency noise was increased by $6 \mathrm{~dB}$. Bebesel et al. [17] achieved BVI noise reductions of up to $5 \mathrm{~dB}$ for different slope angles of a descending Bo 105 helicopter, by featuring a 2/rev IBC strategy during flight tests. Booth and Wilbur [18] emphasized on the acoustic aspects of active rotor blade twist variation, achieving a $2.8 \mathrm{~dB}$ BVI noise reduction with a 5/rev excitation. However, low-frequency noise was increased by up to $5 \mathrm{~dB}$ at an advance ratio of 0.14 .

VRS in conjunction with $\mathrm{ABT}$ offer the potential of improving helicopter rotor performance across a wide range of the flight envelope. Reduction in rotor speed yields reduction in blade profile power, due to lower rotor dynamic head. However, this comes at a cost of increased blade aerodynamic loading arising from the higher collective pitch angles necessary to sustain trim. Increased blade twist results in re-distribution of airloads radially inboards, therefore mitigating induced losses in near-hover conditions. Han et al. [19] presented a combined VRS and ABT numerical approach to improve the performance of a UH-60A helicopter. It was demonstrated that individual variation of rotor speed and blade twist at $250 \mathrm{~km} / \mathrm{h}$ cruise led to $17.8 \%$ and $10.4 \%$ reduction in main rotor power respectively, whilst the combined variation of them raised this figure to 20.9\%. Goulos and Bonesso [20] performed a multi-level assessment of the VRS and ABT concept, including aircraft-engine and mission performance on a Bo 105 helicopter model. A multi-disciplinary optimization approach was deployed to derive globally optimum rotor speed and blade twist schedules towards minimizing engine fuel flow. The devised rotor control schedules were utilized in the simulation of realistic, three-dimensional mission scenarios, showing that benefits associated with the VRS and ABT concepts are mission dependent. Fuel flow and $N O_{x}^{\text {rate }}$ reductions of up to $5 \%$ and $8 \%$ were calculated, respectively. The aeroacoustics of combined VRS/ABT concepts have not yet been investigated. 
In light of the aforementioned research in the existing literature, the scope of this work is to investigate the impact of combined VRS/ABT rotor morphing towards mitigating adverse environmental and acoustic effects associated with descending flight. An integrated framework is developed comprising a series of fundamental modeling methods that address helicopter flight dynamics, engine performance and pollutant emissions prediction, unsteady airloads, rotor aeroelasticity and aeroacoustics. An efficient Design Space Exploration (DSE) and optimization strategy is developed comprising methods for Design of Experiment (DOE), Response Surface Modeling (RSM) and multi-disciplinary optimization for a model representative of the Airbus Helicopters Bo 105 helicopter. The developed framework is deployed to obtain optimal rotor speed and blade twist control schedules, achieving simultaneous reduction of emitted noise and $N O_{x}$ gaseous emissions across a wide range of descending flight states.

\section{Mathematical approach}

To assess the impact of the combined VRS and collective ABT concepts, a holistic modeling approach is followed. A helicopter flight dynamics model calculates the trim solution for the main rotor at a given flight condition. The complex flow field in the vicinity of the main rotor is resolved by a relaxation-based free-wake rotor inflow model, coupled with an unsteady blade aerodynamic model. Noise levels are predicted through an acoustic-analogy-based method. Gaseous emissions prediction is based on stirred-reactor modeling coupled with a zerodimensional engine performance analysis method.

\subsection{Helicopter flight performance model}

The comprehensive framework "HECTOR" (Helicopter Omni-Disciplinary Research Platform) is employed for the calculation of flight dynamics, engine performance and pollutant emissions at aircraft-level for a set of designated operational conditions. HECTOR has been extensively described by Goulos et al. in [21]; [22]; [23]; [24], therefore a brief description of the individual modeling methods will be discussed in this article. 
The rotor blade natural vibration characteristics are obtained using the minimum potential energy method developed by Goulos et al. [21] and extended by Castillo-Pardo et al. [25]. A fifth-order accurate numerical evaluation scheme is employed to calculate the dynamic response of the main rotor blades to encountered aerodynamic or inertial excitations in the time domain. The structural formulation of the rotor model is coupled with the Leishman-Beddoes unsteady nonlinear blade element aerodynamics model [26], the Peters-He finite state induced flow model [27], and the dynamic wake curvature model developed by Goulos [23]. The aforementioned modules are incorporated in a globally-convergent Newton-Raphson method to obtain rotor trim controls and fuselage altitude angles for any designated set of flight conditions.

Gas turbine engine performance is modeled using TURMOMATCH [28], a performance model developed and continuously improved at Cranfield University. TURBOMATCH performs a zerodimensional aero-thermal gas path analysis, solving for the mass and energy balance between the various engine components. For the purpose of this work, the engine Design Point (DP) is set as the maximum contingency power setting. Thus, throughout the part of the operational envelope investigated in this article, the engine operates predominantly at steady-state and Off-Design (OD) conditions. Considering the estimation of $N O_{x}$ gaseous emissions HECTOR incorporates HEPHAESTUS, a generic platform originally developed by Celis et al. [29] for the estimation of pollutant emissions of civil aero-engines. HEPHAESTUS was further developed and extensively validated by Goulos et al. [30] and Ortiz-Carretero [31] to cater for helicopter turboshaft engine $N O_{x}$ gaseous emissions as well. HEPHAESTUS adopts a stirred reactor concept along with a set of simplified chemical reactions, accounting also for the design specifications of the combustion system.

HECTOR and TURBOMATCH establish the aircraft trim state and engine operating point, respectively, for a given set of flight conditions. This process determines, among others, the required rotor thrust $\left(C_{T}\right)$, rolling moment $\left(C_{L}\right)$ and pitching moment $\left(C_{M}\right)$ coefficients. 
Furthermore, $N O_{x}$ gaseous emissions are determined through HEPHAESTUS based on the predefined combustor parameters and the respective inlet conditions. The non-dimensional rotor trim coefficients are then passed to the free-wake model to trim the main rotor and resolve the unsteady airloads necessary for noise predictions.

\subsection{Rotor airloads model}

A free-wake aerodynamic model, developed by Castillo-Pardo [32], is incorporated to cater for high-resolution unsteady airloads. The integrated model comprises a number of methods treating aeroelasticity and aerodynamics. A minimum potential energy method developed by CastilloPardo [25], is deployed for the estimation of coupled flap-lag-torsion vibration characteristics of the elastic rotor blades. The matrix/vector based formulation presented in [22] is employed for modeling the flexible rotor blade kinematics. Leishman-Beddoes indicial response method [26] is utilized towards the prediction of unsteady blade airloads.

A pseudo-implicit predictor-corrector relaxation free-wake method, as proposed by Bagai and Leishman [33], is employed for the modeling of unsteady rotor inflow, able to capture the complex blade-vortex interaction phenomena. The rotor-fuselage aerodynamic interaction is modeled by the semi-empirical analytical formulation proposed by van der Walls et al. [34]. The employed aero-elastic rotor has been developed and validated for the HART-II rotor case [35] in terms of tip-deflections, airloads and structural loads as described in [32]. Rotor and wake discretization as well as the vortex modeling settings are extensively discussed in [36].

The aforementioned models are incorporated in a globally-convergent trim algorithm based on Broyden's numerical algorithm, solving for the rotor collective $\left(\theta_{t w}\right)$ and longitudinal $\left(\theta_{1 S}\right)$ and lateral $\left(\theta_{1 C}\right)$ cyclic controls for given flight conditions with $C_{T}, C_{L}$ and $C_{M}$ coefficients derived by HECTOR. 


\subsection{Rotor aeroacoustics model}

The Integral Computational AeRoacoustics Unified Software (ICARUS) developed by Vouros et al. [36]; [37] has been employed for the aeroacoustic predictions undertaken in this work. An extended description of the model development, applications and validation in terms of acoustic pressure and overall sound pressure level $(O A S P L)$ can be found in [36]. The software is a numerical implementation of Formulation 1A of Farassat [38]; [39], derived as integral solution of the original Ffowcs Williams-Hawkings equation for noise propagation [40]. This employed methodology resolves thickness and loading noise components neglecting the high-speed impulsive noise on the basis that blade tip Mach number does not reach transonic or supersonic values across descending flight conditions. The chord-wise compact thickness noise formulation derived by Lopes [41] is employed for the prediction of thickness noise. Similarly, the chord-wise compact loading noise expression originally proposed by Brentner et al [42] is utilized for the prediction of loading noise, including BVI acoustic components. The use of chord-wise compact acoustic formulations results in significant reductions of the computational overhead enabling the incorporation of physics-based rotor noise predictions into the simulation of complete rotorcraft operations or single-trim flight states.

The acoustic formulations of this framework are compatible with lifting-line-type aerodynamic input provided by the free-wake airloads model. A designated noise hemisphere is employed for the evaluation of rotor source noise. The time-domain acoustic signal is spectrally analyzed via fast Fourier transformation (FFT) to provide a frequency-domain expression of the rotor acoustic impact at the locations of sphere-observers. The FFT signal is integrated in the frequency domain to obtain a single $O A S P L$ value at each designated observer. Broadband noise contribution is then added to the deterministic frequency-domain noise components by employing a semiempirical model developed by Pegg [43]. 


\subsection{Design space exploration}

A dedicated multi-disciplinary optimization framework, hosting the integrated method presented in Sections 2.1-2.3, is developed to obtain optimum rotor speed and blade twist control of the main rotor, leading to concurrent reductions in both noise and $N O_{x}$ gaseous emissions. The optimization algorithm is an expansion of the work presented in [20], implementing multiple objective functions in a multi-disciplinary manner. The method caters for the inherent nonlinearity associated with rotorcraft aerodynamics, aeroelasticity, aeroacoustics and engine combustor performance deploying a computationally efficient strategy capable of evaluating multiple design iterations.

The system's response is evaluated in terms of two objective functions representing specific disciplines of rotorcraft aviation: emitted noise and emitted $N O_{x}$. A cumulative metric of acoustic disturbance representative of the entire acoustic hemisphere is deployed, calculated through energetically averaging the $O A S P L$ over the acoustic hemisphere data points:

$E A O A S P L=10 \log _{10}\left(\frac{1}{N} \sum_{i=1}^{N} 10^{\frac{O A S P L(i)}{10}}\right)$

where EAOASPL is the energetically averaged $O A S P L$ in $\mathrm{dB}$ and $N$ is the number of data points across the surface of the acoustic hemisphere. The EAOASPL metric has been successfully utilized in trajectory optimization for helicopter noise abatement [44]; [45]. The production rate of $N O_{x}$ gaseous emissions $\left(N O_{x}^{\text {rate }}\right)$ constitutes the second objective of the optimization process carried out in this work, to effectively investigate the trade-off between pollutant and noise emissions of helicopters in descent.

A Design Space Exploration (DSE) method is set to identify the system's response to variations of design parameters (variable rotor speed and active blade twist angle) and operational conditions (rotorcraft all-up mass, density altitude, flight speed and climb rate). It comprises two parts; 


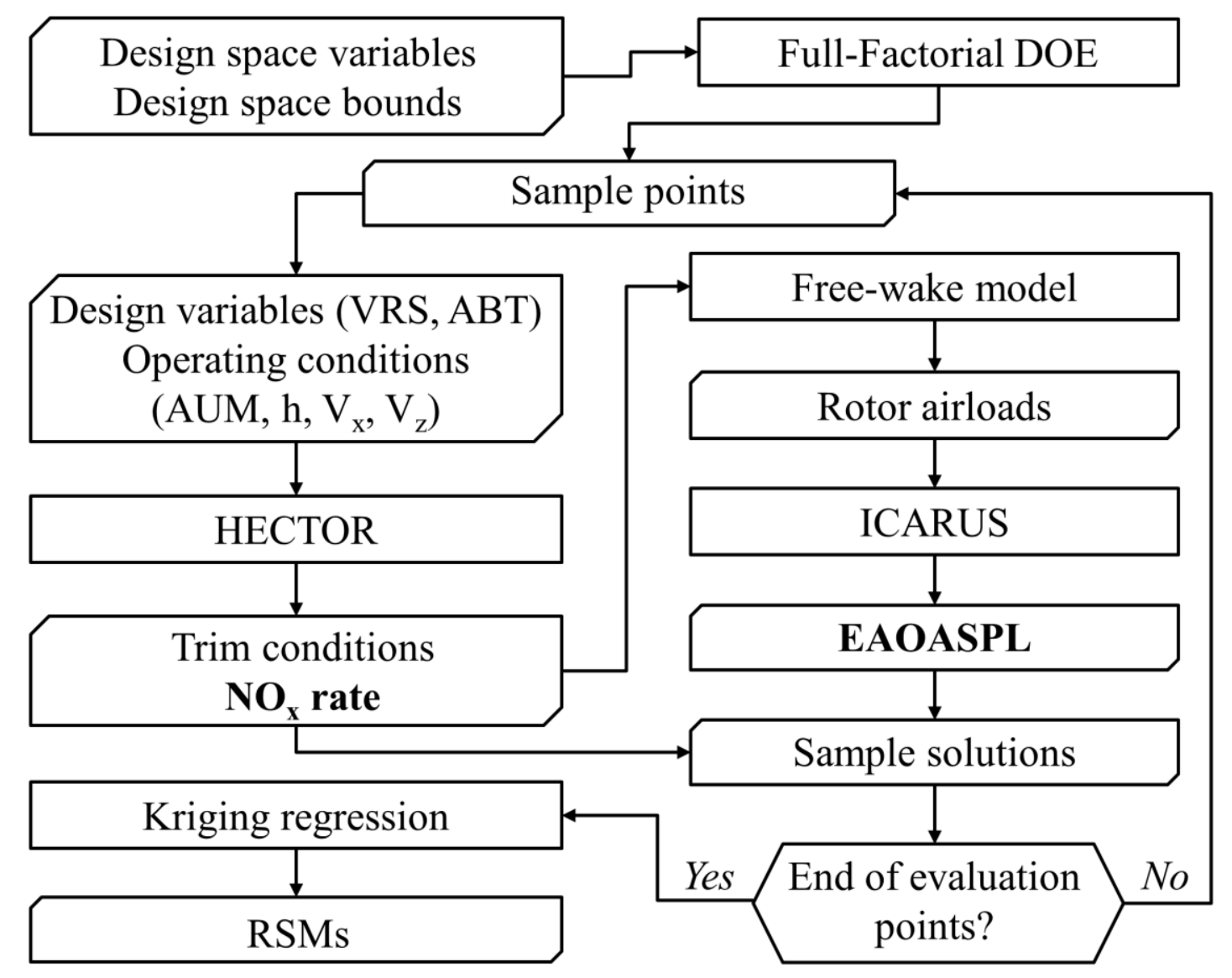

Fig. 1. Overview of Design Space Exploration approach.

(a) the Design of Experiments (DOE) to populate the design space and (b) the construction of Response Surface Models (RSMs) to approximate the system's non-linear response. A DOE is a systematic approach to get the maximum amount of information out of a given sample [20]. A full-factorial design algorithm is implemented to populate the multi-dimensional design space. Factorial sampling allows for different sampling density to be given to each of the design and operational variables based on importance. Additionally, it allows expansion of the design space without distorting the original set of solutions.

Figure 1 presents an overview of the DSE method implemented for the purposes of this work. RSMs are structured using Gaussian Process Regression [46] (Kriging interpolation) across the sample data obtained from the DOE. The purpose of using RSMs instead of direct HECTOR/Free- 
wake/ICARUS runs at each evaluation of the optimization process is to mitigate the excessive computational overhead associated with the multiple simulations related to rotorcraft aeromechanics, aeroacoustics, engine performance and combustor emissions. The predictive accuracy of the derived RSMs is assessed using the classical Leave-One-Out Cross-Validation (LOOCV) method, as described by Kohavi [47].

\subsection{Optimization approach}

After establishing an accurate and computationally efficient DSE approach for the approximation of the non-linear workflow, the available design space was systematically explored to identify optimum VRS and ABT control schedules targeting the concurrent minimization of rotor source noise and $N O_{x}$ gaseous emissions. The optimization algorithm selected for this work is the Nondominated Sorting Genetic Algorithm II (NSGA-II), originally developed by Deb et al. [48]. NSGA-II is an elitist evolutionary algorithm which performs sorting of the population into a hierarchy of non-dominated Pareto fronts. The elite solutions of each generation are directly transformed to the next generation, which prevents back-tracking of the algorithm.

NSGA-II is a global optimization algorithm, which makes the method immune to the danger of getting trapped within design space regions containing local optimum solutions [20]. The final output of the multi-disciplinary optimization process is a Pareto front, which is a set of optimal

solutions. The Pareto-optimal set of solutions is utilized to investigate the optimum trade-off between the multiple objectives of the optimization process. It lies with the designer to select one or more specific point(s) of the Pareto front, based on problem requirements and design reference.

\section{Results and discussion}

A DSE approach comprising full-factorial DOE and Kriging-based RSMs is utilized to approximate the sensitivity of the non-linear system to variations of rotor speed and blade twist. The system's behavior is explored to identify trade-offs between noise and $N O_{x}$ emissions in 
VRS and ABT helicopter configurations. The derived RSMs are deployed towards exporting optimized rotor control schedules across a wide range of descending flight conditions.

\subsection{Surrogate modeling and cross-validation}

The six-dimensional design space is defined by the following design and operational parameters:

(a) variable rotor speed, (b) active blade twist angle, (c) rotorcraft all-up-mass, (d) density altitude,

(e) flight speed and (f) rate of climb. The selected operating parameters are those that govern helicopter rotor noise generation as discussed in [36]. The design space is discretized using 2,916 sample points based on full-factorial sampling design. The sampling grid employs variable sampling density per degree of freedom due to the relative importance of design and operational parameters. The sampling grid structure and design space bounds are provided in Table $\mathbf{1}$. Additionally, reference values have been defined for each operating and design parameter, annotated in parentheses and bold font. The selected reference values represent nominal rotor speed and blade twist in typical descent conditions with a flight path angle of $7.59 \mathrm{deg}$. The threestep integrated procedure described in Section 2.4 is utilized to obtain the values of EAOASPL and $N O_{x}^{\text {rate }}$ for all combinations of parameters annotated in Table 1, using the Bo 105 helicopter model.

Table 1. Design space bounds and values of design and operational parameters to be directly simulated for the DSE process. Reference values annotated in parentheses and bold font.

\begin{tabular}{lll}
\hline Parameter & Values directly simulated & Unit \\
\hline All-up-mass $A U M$ & $1600,(\mathbf{2 0 0 0}), 2400$ & $\mathrm{~kg}$ \\
Density altitude $h$ & $0,(\mathbf{2 5 0}), 500$ & $\mathrm{~m}$ \\
Flight speed $\boldsymbol{V}_{x}$ & $20,(\mathbf{3 0}), 40$ & $\mathrm{~m} / \mathrm{s}$ \\
Climb rate $\boldsymbol{V}_{z}$ & $0,(\mathbf{- 4}),-5$ & $\mathrm{~m} / \mathrm{s}$ \\
Rotor speed $\boldsymbol{\Omega}$ & $75,80,85,90,95,(\mathbf{1 0 0})$ & $\%$ nominal \\
Blade twist angle $\theta_{t w}$ & $2.4,5.2,(\mathbf{8 . 0}), 10.8,13.6,16.40$ & $\mathrm{deg}$ \\
\hline
\end{tabular}


A Kriging-based interpolation is implemented through quadratic regression combined with exponential auto-correlation and a nugget value of $10^{-2}$, to generate the RSMs that efficiently approximate the design space behavior. The Kriging model settings have been selected on the basis of achieving maximum RSM accuracy. The predictive accuracy of the derived RSMs is assessed through Leave-One-Out Cross-Validation (LOOCV) [20]. This method is based on the construction of a dedicated RSM for the design space after excluding a specific sample point from the dataset. The RSM-predicted values of the objective functions at the excluded sample point are then compared to the simulated ones and this process is repeated for every DOE sample point. The quality of the original RSMs is assessed in terms of Pearson's product moment of correlation $N_{\text {Pearson }}[20]$ along with the gradient of the associated linear regression line. A perfectly linear correlation would correspond to $N_{\text {Pearson }}=1.00$ and a regression gradient of $45.00 \mathrm{deg}$.

Figure 2 presents the LOOCV applied to the RSM derived to approximate $N O_{x}^{\text {rate }}$. Very good agreement is observed between the approximated and directly simulated values. Table 2 summarizes the statistical properties of LOOCV for both objectives. Excellent linear correlation is observed for both objectives, with $N_{\text {Pearson }}$ and gradients very close to the ideal values.

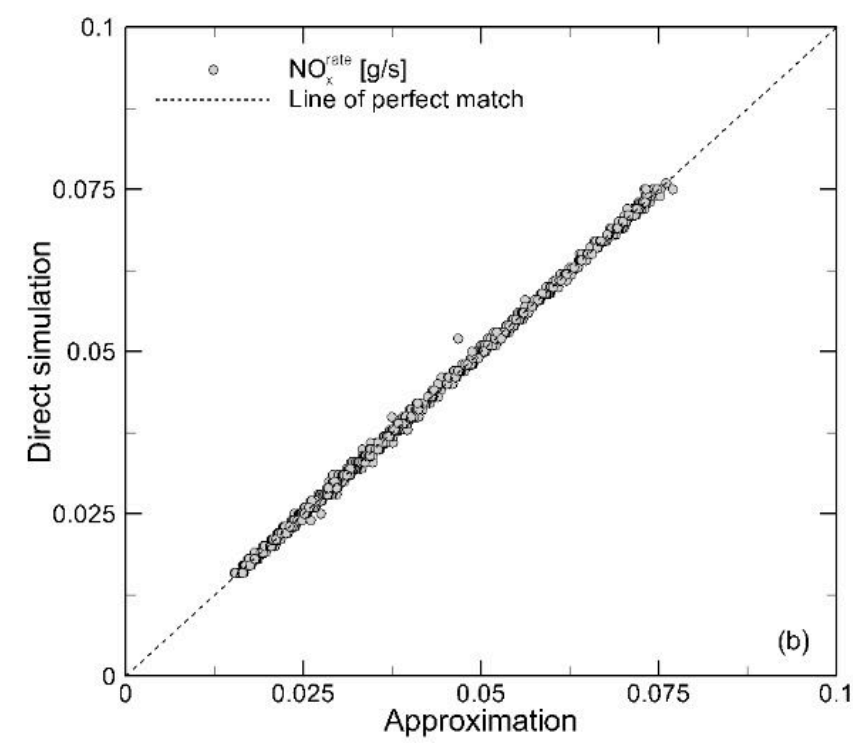

Fig. 2. Leave-one-out Cross Validation applied to the developed RSM for $N O_{x}^{\text {rate }}$ prediction. 
This showcases the ability of the developed RSMs to accurately replicate the physical behavior of the three-step integrated simulation. Furthermore, the normalized root-mean-square error ( NRMSE ) of each objective is of the order of $1 \%$ which quantifies the predictive accuracy of the formulated RSMs. The corresponding standard deviation $\sigma$ of the calculated error is again in the order of $1 \%$ for both variables, indicating that the RSM error is uniformly distributed throughout the investigated design space.

Table 2. Statistical properties of LOOCV for the objectives of interest.

\begin{tabular}{ccccc}
\hline Objective & $\mathbf{N}_{\text {Pearson [-] }}$ & Gradient [deg] & NRMSE [\%] & $\sigma[\%]$ \\
\hline EAOASPL & 0.996 & 44.991 & 0.817 & 0.818 \\
$N O_{x}^{\text {rate }}$ & 0.999 & 45.004 & 1.199 & 1.200 \\
\hline
\end{tabular}

\subsection{Design space behavior}

Having derived accurate RSMs for the objectives under investigation, the behavior of the nonlinear system can be investigated by quantifying the sensitivity of noise and $N O_{x}$ emissions to combined variations of rotor speed $(\Omega)$ and blade twist $\left(\theta_{t w}\right)$ throughout the helicopter operational envelope for descending flight. Relative metrics have been adopted for the quantifications of this work. Specifically, $\Delta N O_{x}^{\text {rate }}[\%]=100 \cdot \frac{N O_{x}^{\text {rate, } \text { Opt }}-N O_{x}^{\text {rate }, \text { Nom }}}{N O_{x}^{\text {rate }, \text { Nom }}}$ represents the normalized change of $N O_{x}^{\text {rate }}$ with respect to the corresponding value at nominal $\Omega$ and $\theta_{t w}$. The dimensional values in noise reduction were preferred from normalized ones, since this is the convention typically used in noise abatement studies. Therefore, $\triangle E A O A S P L=\left(E A O A S P L^{O p t}-E A O A S P L^{N o m}\right)$ represents the difference in dB relative to nominal rotor control settings at the specified flight conditions. 

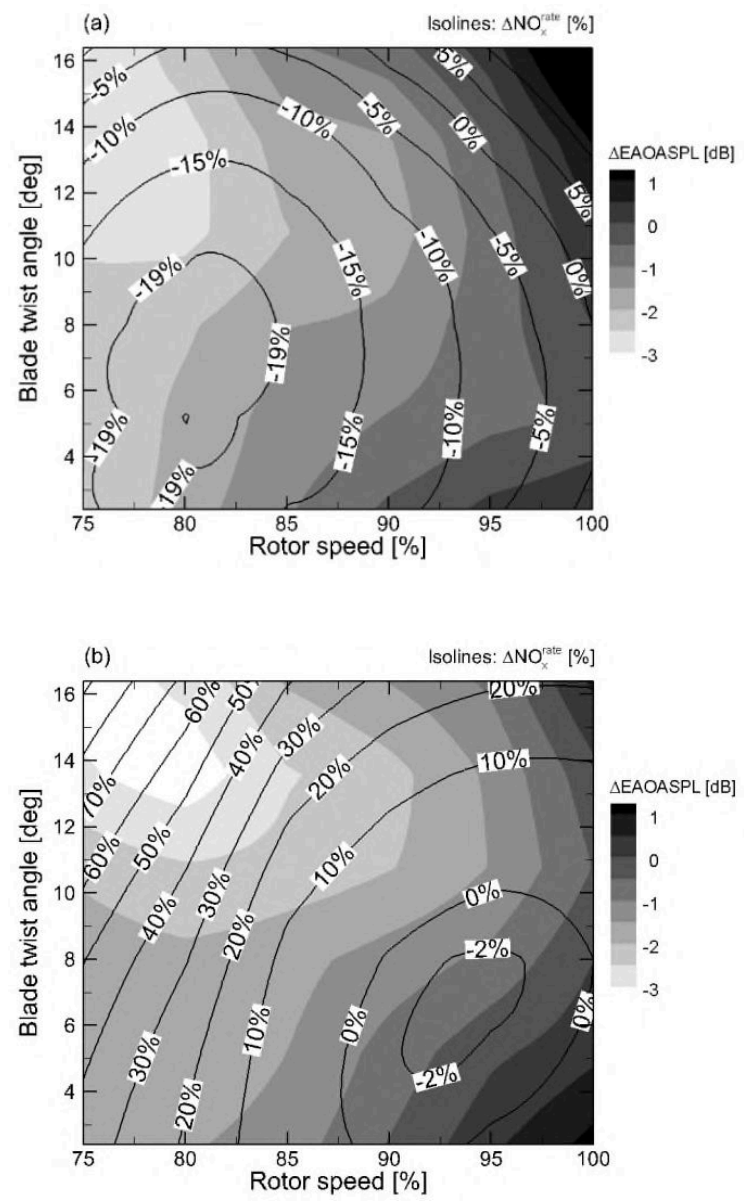

Fig. 3. Design Space Behavior: impact of all-up mass ( $A U M$ ) on combined variable rotor speed and active blade twist noise and $N O_{x}$ emissions at $h=250 \mathrm{~m} V x=30 \mathrm{~m} / \mathrm{s}$ and $V z=-4 \mathrm{~m} / \mathrm{s}:$ (a) $A U M=1600 \mathrm{~kg}$, (b) $A U M=2400 \mathrm{~kg}$.

Figure 3 (a) demonstrates the impact of VRS and ABT variation on $\triangle E A O A S P L$ and $\Delta N O_{x}^{\text {rate }}[\%]$ at $A U M=1600 \mathrm{~kg}$. The rest of the flight conditions $h, V_{x}$ and $V_{z}$ retain their reference values as described in Table 1. The noise contours depict that EAOASPL is minimum at low $\Omega$ and high $\theta_{t w}$ at these flight conditions. From an aeroacoustic perspective, reduced $\Omega$ yields reduced relative Mach numbers which eventually lead to reduced acoustic radiation Mach numbers. This results in a lower Doppler amplification factor which, according to the derivations of thickness and loading noise in Formulation 1A of Farassat, mitigates the 


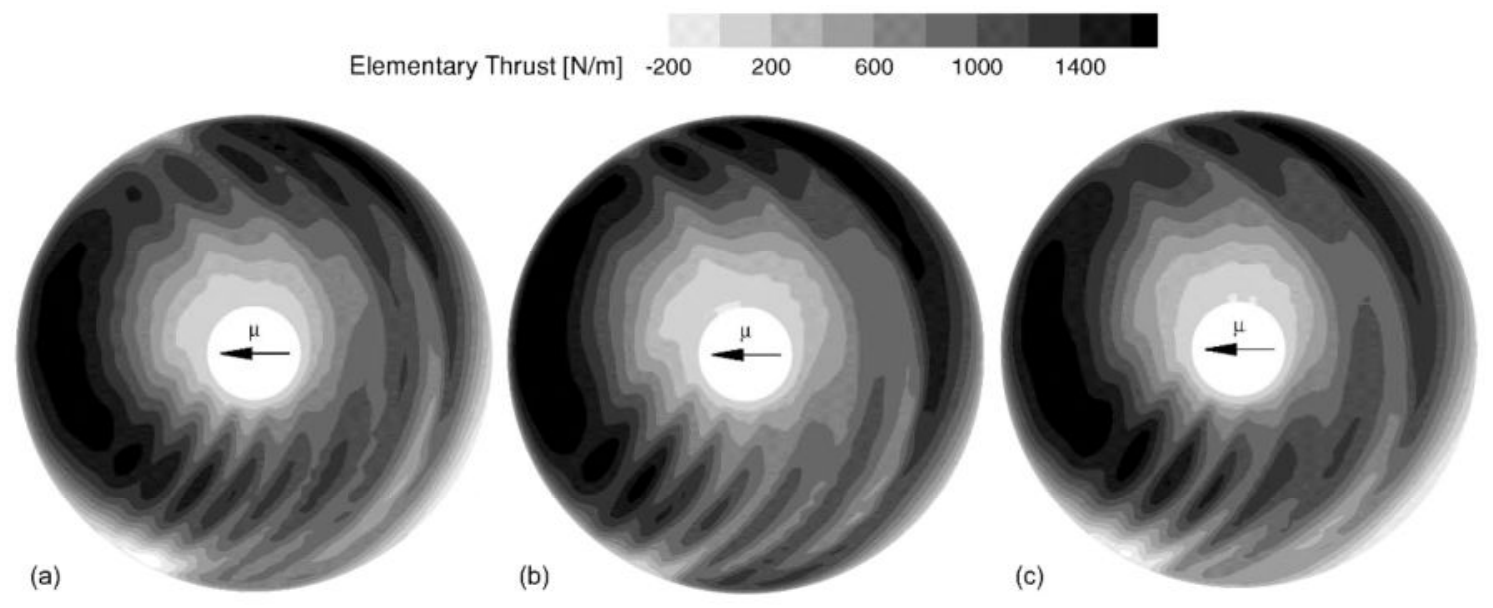

Fig. 4. Distribution of elementary thrust across the rotor disc of Bo 105 helicopter with $V_{x}=30 \mathrm{~m} / \mathrm{s}, V_{z}=-4 \mathrm{~m} / \mathrm{s}, A U M=1600 \mathrm{~kg}$ and $h=250 \mathrm{~m}:$ (a) $\Omega=100 \%$, $\theta_{t w}=8 \mathrm{deg}$; (b) $\Omega=75 \%$ and $\theta_{t w}=8 \mathrm{deg}$ and (c) $\Omega=75 \%$ and $\theta_{t w}=13.6 \mathrm{deg}$.

relative contribution of the acoustic terms of both deterministic noise components. Variation in $\theta_{t w}$ leads to a re-distribution of aerodynamic loading across the rotor disc. Specifically, increased $\theta_{t w}$ tends to move the peaks of blade loading radially inwards. As discussed in [35], the most severe BVIs occur at azimuthal angles around $\psi=50 \mathrm{deg}$ and $\psi=300 \mathrm{deg}$, mainly focused at the outboard regions of the blades, i.e. towards the blade tips. This is due to the small missdistance between trailed tip-vortices and rotor blades around the aforementioned regions. Hence, re-distribution of aerodynamic blade loading towards the inboard direction of the blade span leads to reduced circulation around the tip which reduces tip-vortex strength and eventually BVI noise, as also shown in Fig. 4.

In Fig. 3 (a), the $\Delta N O_{x}^{r a t e}[\%]$ isolines are superimposed to illustrate the trade-off between noise and $N O_{x}$ emissions under variations in $\Omega$ and $\theta_{t w}$. The production of $N O_{x}$ emissions is dependent on the engine operating point which is determined by two fundamental disciplines: (a) rotor aerodynamic performance and (b) engine thermodynamic performance. In terms of main 
rotor aerodynamics and performance, reduction of $\Omega$ results to a reduced local velocity magnitude and a lower rotor dynamic head. Consequently, the rotor blade profile drag will be reduced [49]. However, a simultaneous raise in blade local pitch angle by collective pitch is necessary to restore rotor thrust to meet trim requirements. This inevitably leads to a higher local angle of attack which imposes an increased blade loading [20]. It is noted that excessive increase in blade loading can negatively affect the aerodynamic performance of rotor blade elements and eventually, increase rotor power requirements. As regards engine performance, Walsh and Fletcher [50] showed that at a given rotational speed of the gas generator (GG), there is a unique free-power turbine (FPT) speed that minimizes specific fuel flow $(S F C)$ and maximizes power generation, as also shown by Goulos and Bonesso [20]. Hence, it is the trade-off between engine thermodynamic deficit and rotor aerodynamic benefit which determines the overall impact of VRS on fuel consumption and $\mathrm{NO}_{\mathrm{x}}$.

Fig. 3 (b) presents the corresponding behavior of $\triangle E A O A S P L$ and $\triangle N O_{x}^{r a t e}[\%]$ at $A U M=2400 \mathrm{~kg}$ and reference flight conditions as described in Table 1. It can be seen that $\Omega$ higher from the corresponding value of the $A U M=1600 \mathrm{~kg}$ case is required to achieve $N O_{x}^{\text {rate }}$ reductions. A lower $\Omega$ in this case would result in a very high rotor collective pitch angle to sustain trim, at a cost of overly increased aerodynamic loading and consequently, power requirement and $N O_{x}$ emissions. At high $A U M$, reductions in $\Omega$ result in both aerodynamic and thermodynamic performance deficits, which give a steep gradient in $N O_{x}$ production. This is in accordance with the findings reported in Refs. [20]; [49]; [51], which indicate higher performance benefits at "low-and-light" flight conditions. As regards noise, the minimum $\Omega$ is required due to Doppler amplification effects, whilst a higher value of $\theta_{t w}$ is required to compensate for the increased blade loading associated with high $A U M$. 


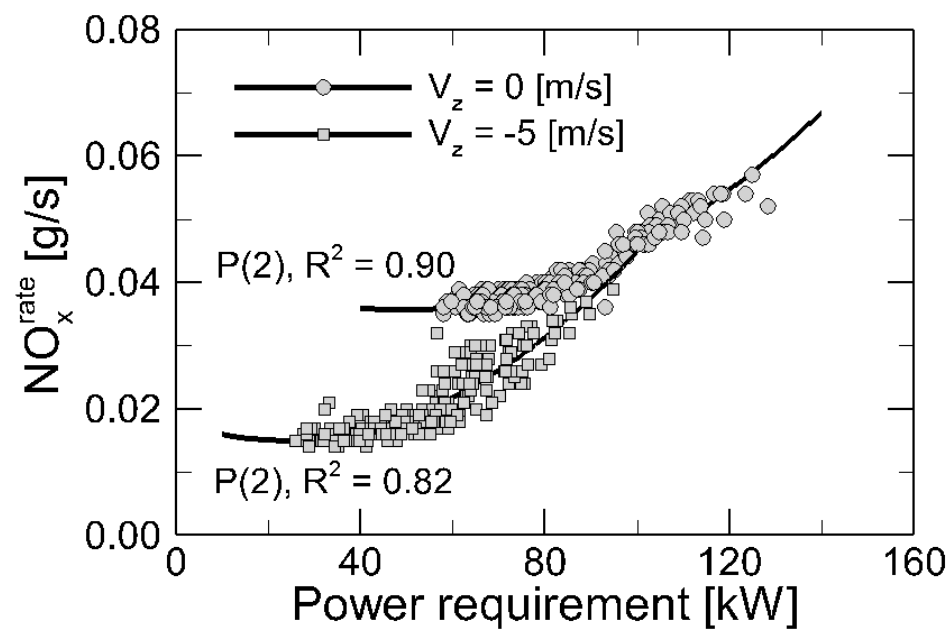

Fig. 5. Correlation between rotor power requirement and $N O_{x}$ rate for $V_{z}=O \mathrm{~m} / \mathrm{s}$ $V_{z}=-5 \mathrm{~m} / \mathrm{s}$ at $A U M=1600 \mathrm{~kg}$.

Figure 5 presents the correlation between rotor power requirement and $N O_{x}$ emissions. It is shown that families of correlated points are formed using a $2^{\text {nd }}$ order polynomial regression with coefficients of determination $R^{2}=0.90$ and $R^{2}=0.82$ for $V_{z}=0 \mathrm{~m} / \mathrm{s}$ and $V_{z}=-5 \mathrm{~m} / \mathrm{s}$, respectively. These refer to $A U M=1600 \mathrm{~kg}$, but similar response was found for the rest AUMs. The associated amplitude-normalized standard deviations are $13.26 \%$ and $25.33 \%$, due to the impact of the engine thermodynamic performance. However, the monotonically-ascending trend between the two variables suggests that the main driving factor for $N O_{x}$ generation is rotor aerodynamic performance, therefore $N O_{x}$ is used as surrogate for rotor power in the analysis to follow. Variations in $\theta_{t w}$ primarily affect rotor induced power which dominates the low-speed region of the flight envelope. At low-speed conditions, an increase in $\theta_{t w}$ provides a more uniform inflow through the re-distribution of radial blade circulation, which leads to reduced induced losses. 


\subsection{Trade-off between source noise and NOx emissions}

The analysis of design space behavior highlighted the potential of combined VRS and ABT rotor morphing in reducing the EAOASPL emissions and $N O_{x}^{\text {rate }}$, as well as depicted the strong trade-offs between the two aforementioned objectives. These highlight the potential of multidisciplinary optimization, targeting the simultaneous mitigation of acoustic and environmental impact of descending helicopters. The NSGA-II-based optimization strategy introduced in Section 2.5 is deployed for the export of Pareto optimal fronts in a parametric manner, as function of the governing operational parameters $V_{x}, V_{z}, A U M$ and $h$. A maximum of 40 generations with a population of 40 for each generation has been found sufficient for the convergence of the optimizer. The scope of this parametric investigation is to identify trade-offs and sensitivities between the conflicting metrics of interest: $\triangle E A O A S P L$ and $\triangle N O_{x}^{\text {rate }}[\%]$.

Figure 6 (a) illustrates the Pareto optimal fronts corresponding to five different $A U M$ values, ranging from $A U M=1600 \mathrm{~kg}$ to $A U M=2400 \mathrm{~kg}$. The rest of the operating parameters have their reference values as presented in Table 1. It is observed that the highest noise benefits can be achieved at high $A U M$. On a highly loaded rotor, circulation peaks are located closer to the blade tips, compared with a lightly loaded rotor [52]. Hence, collective ABT can more efficiently off-load the tips by moving the circulation peaks inboards therefore reducing tip-vortex intensity and eventually BVI noise. Regarding $N O_{x}^{\text {rate }}$, it is noticed that the greatest reductions are achieved at low $A U M$, as discussed in Fig. 3 (a). At high $A U M$, no significant benefits can be achieved due to the limited reduction margins for $\Omega$. Under such conditions, a reduced rotor speed would require a considerable increase in rotor collective pitch angle to sustain trim, which would impose excessive aerodynamic performance penalties. 

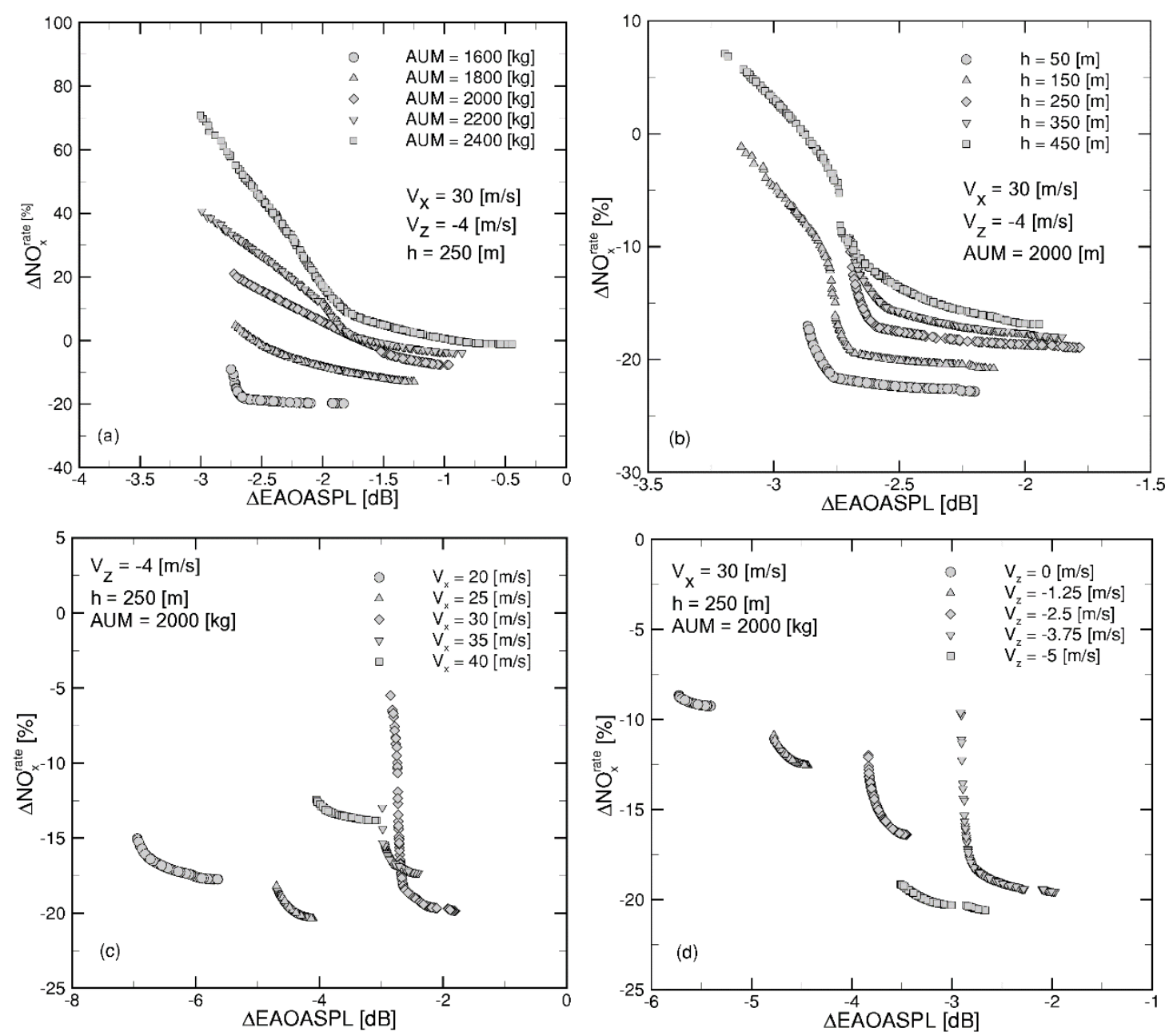

Fig. 6. Impact of operational variables on Pareto fronts of optimized combined $\Omega$ and $\theta_{t w}$ helicopter rotor; (a) all-up mass $A U M$, (b) density altitude $h$, (c) flight speed $V_{x}$ and (d) climb rate $V_{z}$

Figure 6 (b) presents the Pareto fronts corresponding to a series of density altitudes ranging from $h=50 \mathrm{~m}$ to $h=450 \mathrm{~m}$. It is shown that $N O_{x}$ optimal solutions are significantly affected by altitude variations, offering up to $22.53 \%$ reductions in $N O_{x}^{\text {rate }}$. At a higher altitude, the reduced air density leads to lower elementary blade lift which in turn results in a higher rotor power requirement. This imposes limited margins with respect to beneficial rotor speed reduction. Therefore, at higher altitudes, $N O_{x}$-related benefits from combined VRS/ABT are smaller 
compared to low-altitude conditions. On the other hand, EAOASPL is not considerably altered in the investigated range of density altitude variations.

Figure 6 (c) addresses the impact of horizontal flight speed $\left(V_{x}\right)$ on the trade-off between $\triangle E A O A S P L$ and $\triangle N O_{x}^{\text {rate }}[\%]$ for VRS and ABT rotors. The highest EAOASPL benefits are achieved in low flight speed for this specific climb rate and can be attributed to the impact of ABT in this speed regime. Specifically, the combination of $V_{x}=20 \mathrm{~m} / \mathrm{s}$ with the reference climb rate $V_{z}=-4 \mathrm{~m} / \mathrm{s}$ leads to a considerable increase in radially inboards airloads due to the impact of the vertical free-stream velocity component. Collective ABT can effectively mitigate these effects by reducing $\theta_{t w}$ to values lower than the nominal.

As regards $N O_{x}$, relatively "flat" Pareto fronts are obtained for most $V_{x}$ settings, due to the shape of the power curve in this flight speed regime [52]. The variation of rotor power requirements as function of flight speed follows a "bucket" shape, with zero gradient between $V_{x}=20 \mathrm{~m} / \mathrm{s}$ and $V_{x}=30 \mathrm{~m} / \mathrm{s}$ for this specific helicopter. This is reflected on the sensitivity of $\Delta N O_{x}^{r a t e}[\%]$ to $V_{x}$ variations, as discussed in this investigation. However, a considerably wide range in potential $N O_{x}$ improvements, as well as non-monotonicity with respect to $V_{x}$, is observed at $V_{x}=30 \mathrm{~m} / \mathrm{s}$. Considering the reference climb rate $V_{z}=-4 \mathrm{~m} / \mathrm{s}$ and the $3 \mathrm{deg}$. longitudinal tilt of the Bo 105 rotor shaft, an equivalent "wind tunnel" shaft angle of $a_{s}=-4.59 \mathrm{deg}$ would occur. This is the angle that is most sensitive to BVI phenomena because of the small miss-distance between the blades and tip-vortices [35]. Hence, variations of $\Omega$ and $\theta_{t w}$ have a strong impact on tip vortex formation and consequently, rotor performance and $N O_{x}$ emissions. 
Similar trends are shown in Fig. $6(\mathbf{d})$, which presents the impact of climb rate $\left(V_{z}\right)$ on the optimum trade-off between $\triangle E A O A S P L$ and $\triangle N O_{x}^{\text {rate }}[\%]$ for VRS and ABT rotors. A wide range of $N O_{x}^{\text {rate }}$ reductions is observed at $V_{z}=-3.75 \mathrm{~m} / \mathrm{s}$, which replicates the strong BVI conditions of the HART II experiment [35]. The highest $\Delta N O_{x}^{\text {rate }}[\%]$ benefits are obtained at $V_{z}=-5 \mathrm{~m} / \mathrm{s}$, which represents the most lightly loaded condition for the rotor in the examined flight envelope. Profile power becomes progressively significant for those conditions, hence there is substantially more margin to reduce power requirement and therefore $N O_{x}$. The highest $\triangle E A O A S P L$ reductions are achieved at level-flight $\left(V_{z}=0 \mathrm{~m} / \mathrm{s}\right)$. In low-speed level-flight, the main rotor vortical wake encounters strong roll-up leading to the formation of a pair of intense trailing vortices let from the sides of the rotor, similar to the wing tip vortices generated by fixedwing aircraft. The concentration of strong vortical structures at the two sides of the rotor results in the formation of two strong loading cells at rotor azimuth angles $\psi=80 \mathrm{deg}$ and $\psi=280 \mathrm{deg}$ located in the blade tip region. These essentially constitute significant sources of loading noise, which is effectively mitigated with collective ABT, by moving the circulation radial peaks towards the inboard rotor direction, which finally alters the tip vortex circulation and therefore the intensity of BVI events.

\subsection{Optimized rotor control schedules}

Having established a methodology for the generation of Pareto optimal fronts for $\triangle E A O A S P L$ and $\Delta N O_{x}^{r a t e}[\%]$, one or more specific points from the Pareto fronts can be selected based on design intent. In this work, three points will be selected representing: (a) minimum rotor noise, (b) best trade-off between noise and $N O_{x}$ emissions, and (c) minimum $N O_{x}$ emissions. The Pareto point which corresponds to minimum noise emissions is essentially the point that achieves 
minimum (most negative) $\triangle E A O A S P L$. Similarly, the minimum $N O_{x}$ point is the Pareto point which achieves minimum (most negative) $\Delta N O_{x}^{r a t e}[\%]$. The minimum Euclidean distance selection method [53] is utilized to determine the Knee point of the Pareto front which will serve as trade-off point for the multi-disciplinary optimization. Specifically, a Utopia-optimal point is determined as the combination of minimum noise and minimum $N O_{x}$ points. This is a fictitious optimal point which would achieve idealized minimization of both objectives, concurrently. The Pareto trade-off point is then determined as the point which has the minimum Euclidean distance from the Utopia-optimal point. It is noted that for the calculation of Euclidean distance of each Pareto point from the Utopia-optimal point, the front is previously normalized (\%) in both dimensions. In this work, the aforementioned optimal selection strategy has been generalized to export optimized VRS and ABT control schedules for noise minimization, $N O_{x}$ minimization and a trade-off between these two objectives. This is selected as an indicative solution that corresponds to the best trade-off between the two conflicting performance metrics of interest.

Figure 7 (a)-(f) presents the optimized rotor control schedules exported using the optimization method presented in Section 2.5. The schedules refer to $A U M=1600 \mathrm{~kg}$ at a reference density altitude $h=250 \mathrm{~m}$. The provided schedules cover the entire horizontal speed $\left(V_{x}\right)$ and climb rate $\left(V_{z}\right)$ range of the descending flight envelope of twin-engine light helicopters. For each and every point of the schedules, a dedicated multi-disciplinary optimization is carried out, generating a unique Pareto front. Subsequently, the minimum noise, trade-off and minimum $N O_{x}$ points are extracted. As regards noise optimization, it can be seen that the optimal rotor speed $\Omega^{O p t}$ is generally lower at low $V_{x}$. Values below $80 \%$ are favored to benefit from the Doppler amplification factor effects, which reduce the relative impact of all loading noise components. Higher $\Omega^{O p t}$ is favored for $N O_{x}$ optimization to prevent any adverse effects on 

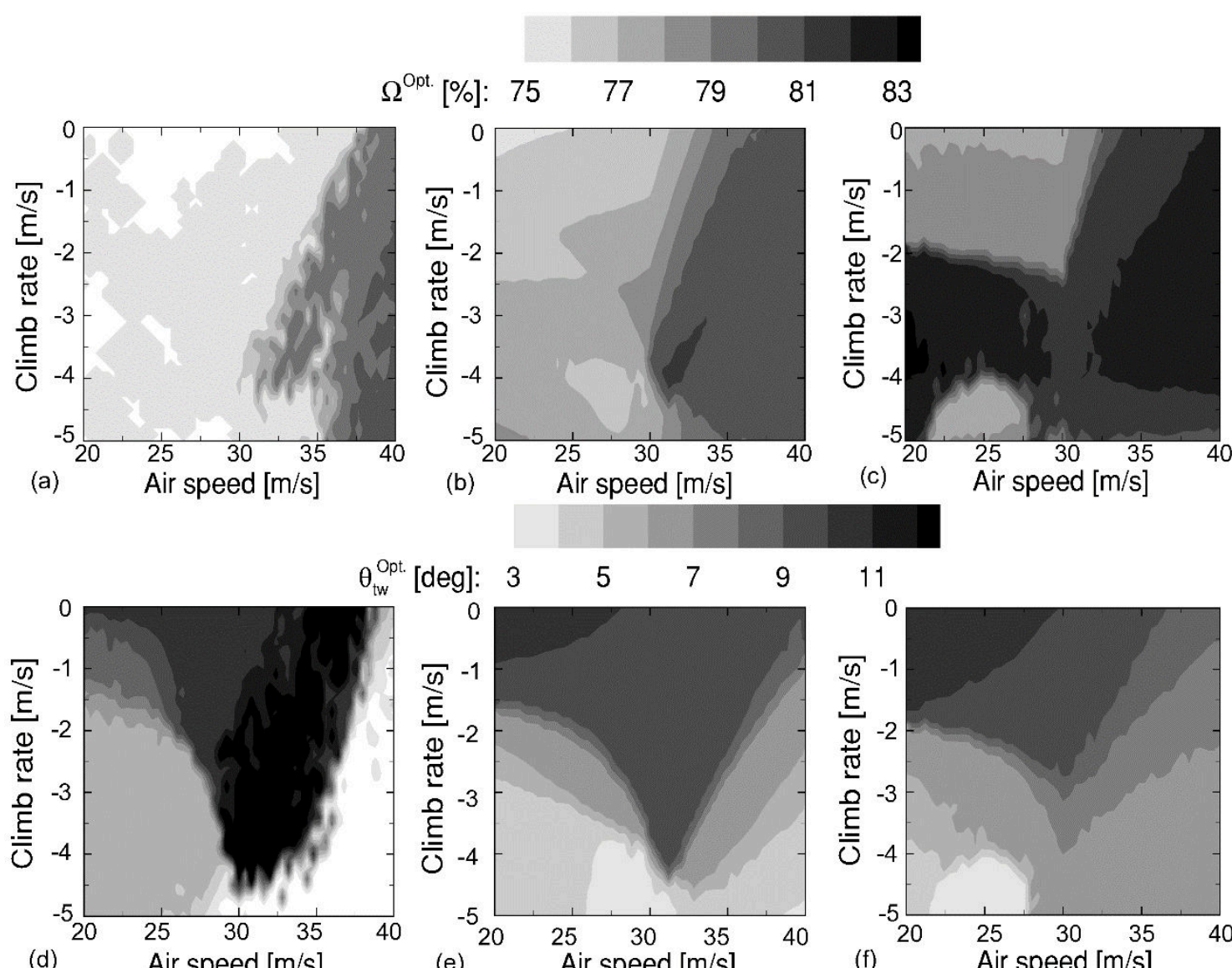

(b)

$\theta_{\mathrm{tw}}^{\text {Opt. }}[\mathrm{deg}]: \begin{array}{lllll}3 & 5 & 7 & 9 & 11\end{array}$

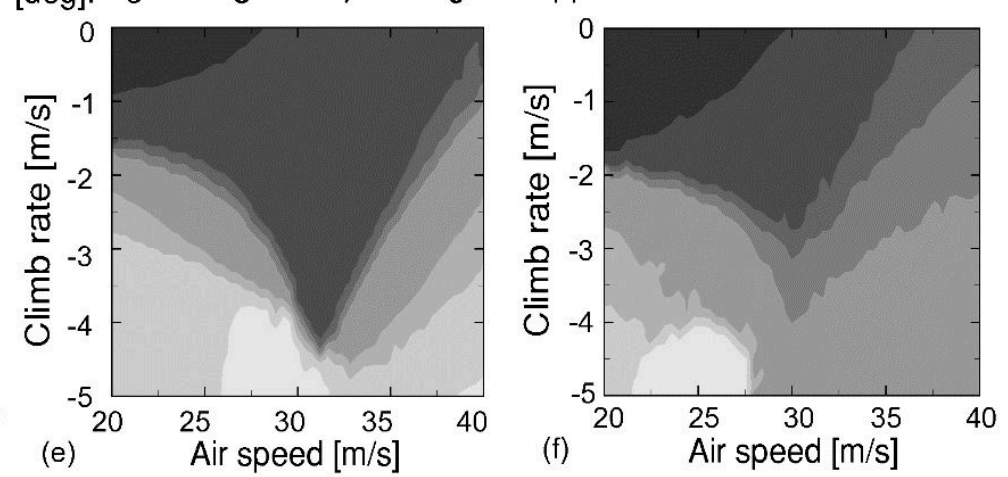

Fig. 7. Optimized rotor VRS and ABT schedules for $A U M=1600 \mathrm{~kg}$ : Optimal rotor speed for (a) EAOASPL, (b) best trade-off, (c) $N O_{x}$; Optimal blade twist angle for (d) EAOASPL , (e) best trade-off, (f) $N O_{x}$.

rotor power that would otherwise be induced by the low blade dynamic head and increased rotor blade loading to sustain trimmed flight.

As regards optimal blade twist angle $\theta_{t w}^{O p t}$, generally high blade twist angles (above $11 \mathrm{deg}$ ) are preferred to minimize noise in the regions of the flight envelope where the aerodynamic behavior of the main rotor is dominated by BVI events. This is due to the impact of blade twist that tends to bias the blade loading radially inwards, thus reducing the strength of the rotor tip vortices and the concurrent intensity of BVI noise. In the rest of the descending region though, $\theta_{t w}^{O p t}$ is lower 
than the nominal $\theta_{t w}$ to compensate for the increased inwards blade loading associated with high free-stream upwash $\left(V_{z}\right)$.

In terms of $N O_{x}$ minimization, high $\theta_{t w}^{O p t}$ is required in the low-speed region, indicating the effectiveness of radial circulation redistribution in increasing rotor aerodynamic efficiency in the low-speed region. At $V_{z}=-5 \mathrm{~m} / \mathrm{s}$, a "pocket-shaped" region of reduced $\theta_{t w}^{O p t}$ requirement is observed around the flight speed of max endurance, $V_{x}=25 \mathrm{~m} / \mathrm{s}$, corresponding to this specific descent rate, which is attributed to the reduced rotor power requirements. This is also reflected in the distribution of $\Omega^{O p t}$, as shown in Fig. 7 (c). The optimized $\Omega$ and $\theta_{t w}$ schedules that achieve the best trade-off between noise and $N O_{x}$ essentially facilitate optimal control patterns from both disciplines.

Figure 8 (a)-(f) quantifies the EAOASPL and $N O_{x}^{\text {rate }}$ benefits attainable by the derived control schedules at $A U M=1600 \mathrm{~kg}$. The highest $N O_{x}$ benefits are expected when power requirements are low, hence at low $V_{x}$ and $V_{z}$. For $\triangle E A O A S P L$, deltas up to $-7 \mathrm{~dB}$ are observed in steep descent, due to the effectiveness of collective ABT in re-distributing aerodynamic loading resulting from the strong vertical free-stream velocity component. Additionally, considerable noise reduction is achieved in level flight due to the mitigation of Doppler amplification effects through VRS, combined with the reduction of vortex roll-up effects achieved through blade tip off-loading due to collective ABT. The former mechanism yields reduced thickness noise contribution, whilst the latter mitigates loading noise. The smallest, but still significant, noise reductions are located in the BVI-dominated region of the flight envelope, around $V_{x}=32.5 \mathrm{~m} / \mathrm{s}$ and $V_{z}=-4 \mathrm{~m} / \mathrm{s}$. This indicates that the combined VRS and ABT rotor morphing concept cannot entirely diminish the impact of intensive BVIs, because it does not include higher harmonic blade pitch control. Nevertheless, the proposed concept is capable of 
$\triangle$ EAOASPL [dB]: $\quad \begin{array}{lllll}-7 & -6 & -5 & -4 & -3\end{array}$

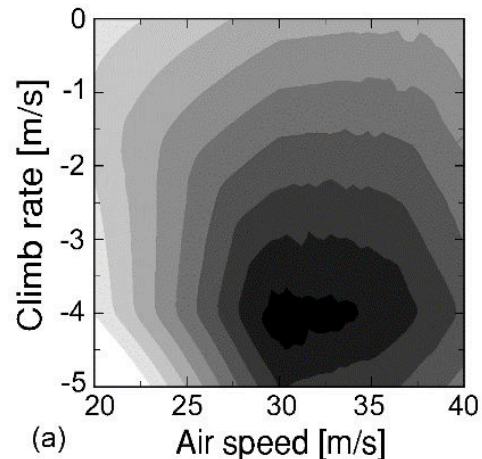

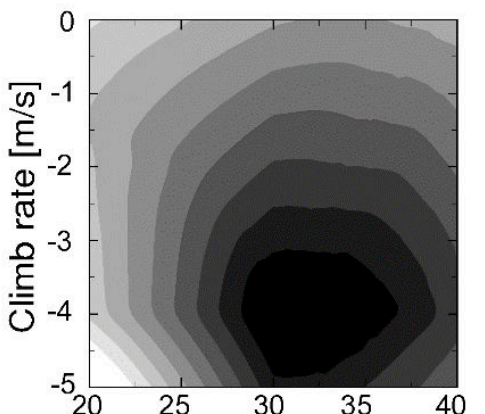

(b) $\quad$ Air speed $[\mathrm{m} / \mathrm{s}]$

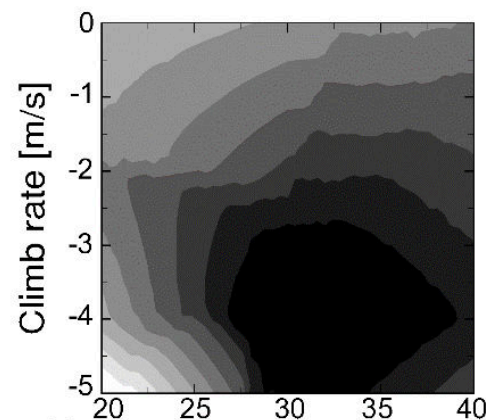

(c) $\quad$ Air speed $[\mathrm{m} / \mathrm{s}]$

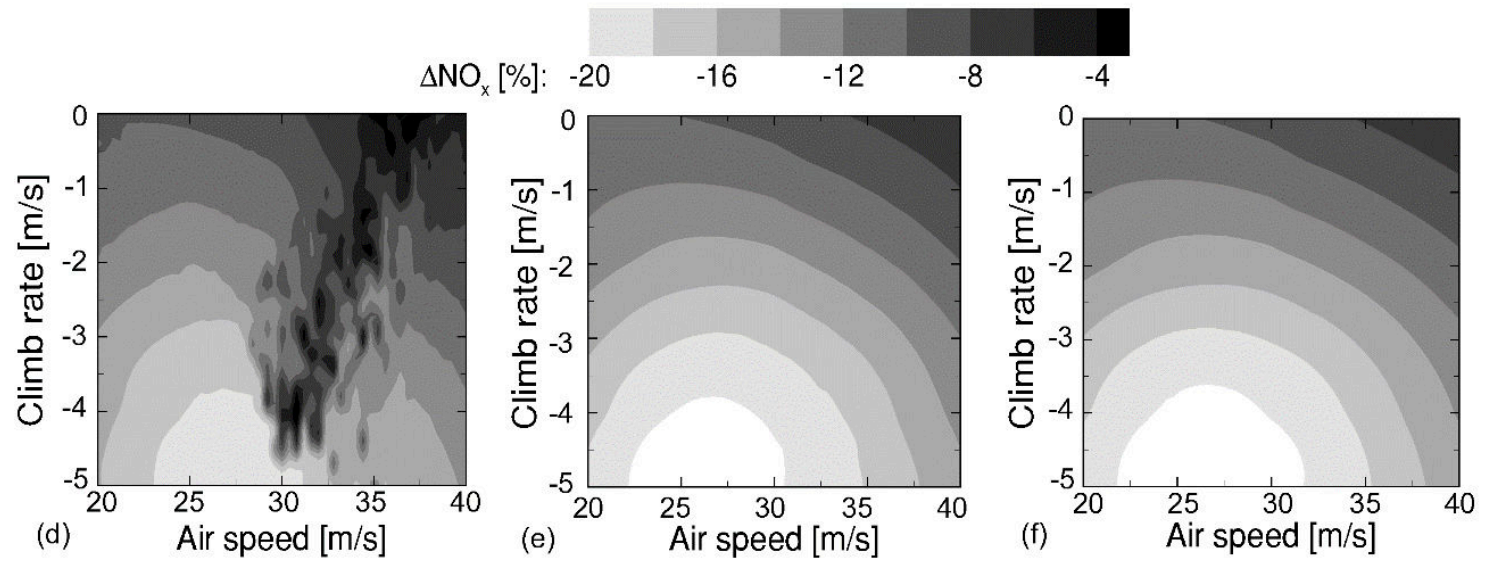

Fig. 8. EAOASPL and $N O_{x}$ benefits for $A U M=1600 \mathrm{~kg}$ : Noise benefits through optimizing for (a) EAOASPL, (b) best trade-off, (c) $N O_{x} ; N O_{x}$ benefits through optimizing for (d) EAOASPL, (e) best trade-off, (f) $N O_{x}$.

achieving overall noise reductions in the range of 2.31 to $8.30 \mathrm{~dB}$. Additionally, the acquired EAOASPL benefits come with concurrent $N O_{x}^{\text {rate }}$ reductions ranging from $-6.42 \%$ to $-21.32 \%$ when the trade-off schedules are applied.

Figure 9 (a)-(f) presents the $\Omega^{O p t}$ and $\theta_{t w}^{O p t}$ schedules targeting at minimum noise, best tradeoff and minimum $N O_{x}$ emissions, respectively, for $A U M=2000 \mathrm{~kg}$. The observed patterns are similar to the $A U M=1600 \mathrm{~kg}$ case, albeit suggesting higher $\Omega^{O p t}$ and $\theta_{t w}^{O p t}$ settings due to the higher thrust requirement compared to the $A U M=1600 \mathrm{~kg}$ case. The 


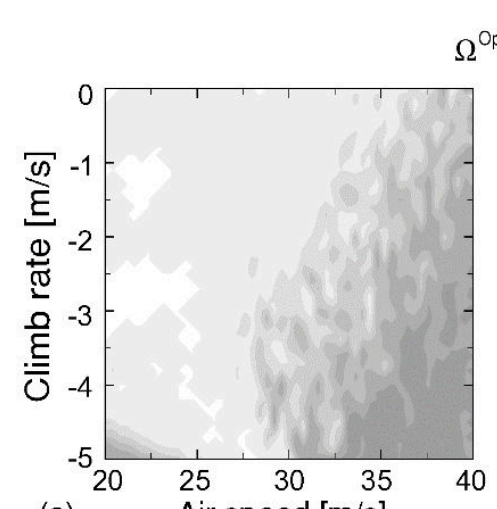

(a) Air speed $[\mathrm{m} / \mathrm{s}]$

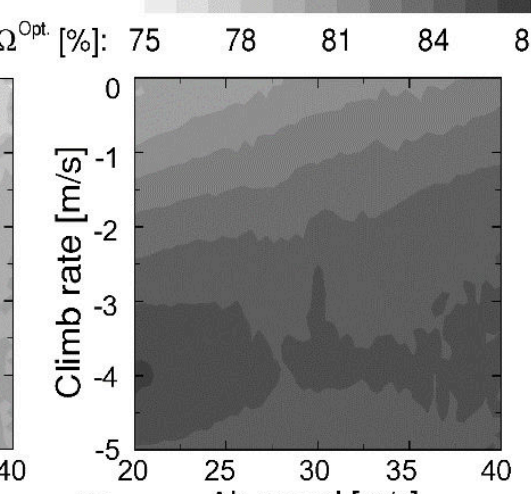

(b) Air speed $[\mathrm{m} / \mathrm{s}]$

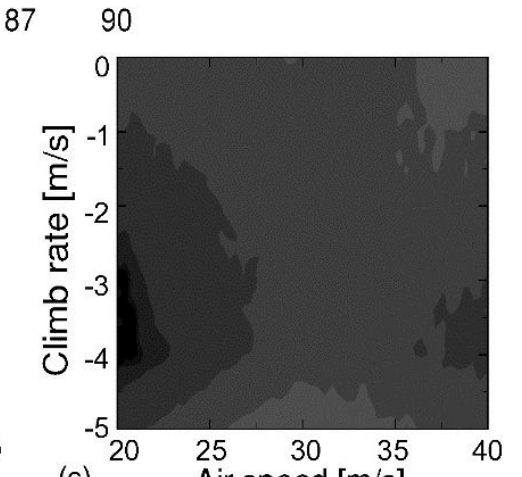

(c)

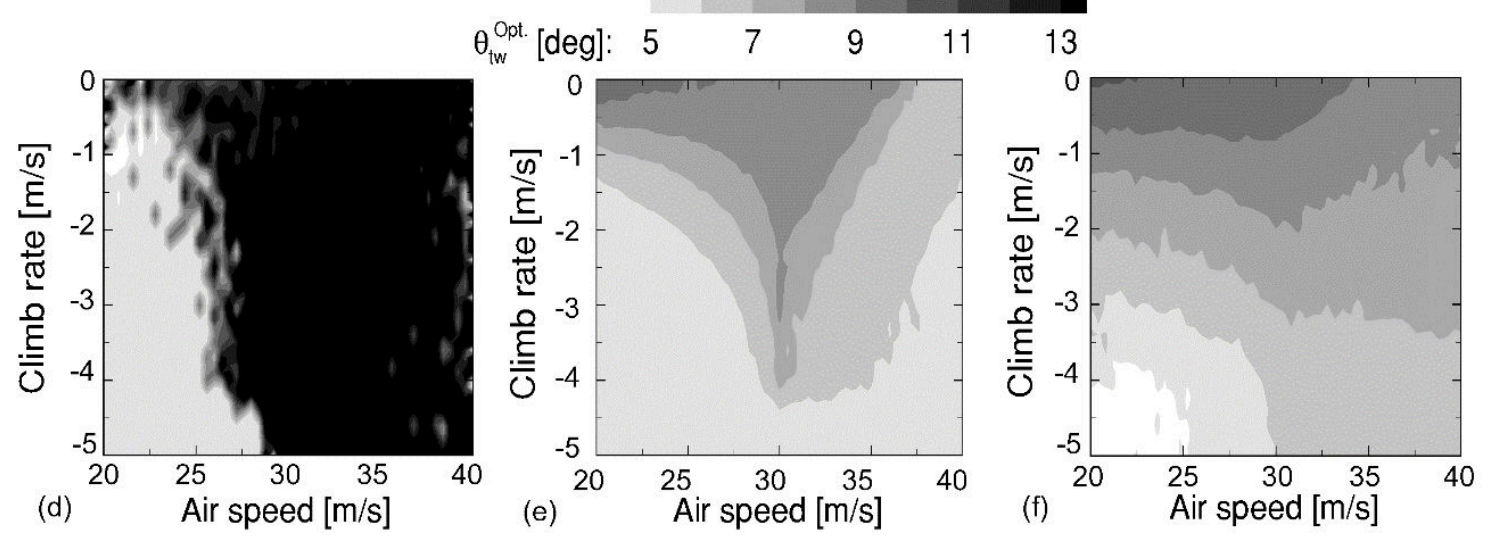

Fig. 9. Optimized rotor VRS and ABT schedules for $A U M=2000 \mathrm{~kg}$ : Optimal rotor speed for (a) $E A O A S P L$, (b) best trade-off, (c) $N O_{x}$; Optimal blade twist angle for (d) $E A O A S P L$ , (e) best trade-off, (f) $N O_{x}$.

corresponding EAOASPL and $N O_{x}^{\text {rate }}$ benefits for $A U M=2000 \mathrm{~kg}$ are provided in Fig. 10 (a)-(f). The noise-optimized schedules offer noise reductions up to $-6.62 \mathrm{~dB}$; however, this comes at a $N O_{x}$ increase cost, even up to $60 \%$. The $N O_{x}$-optimized schedules provide $N O_{x}$ benefits up to $\Delta N O_{x}^{\text {rate }}=-10.29 \%$ along with noise reductions between 1.07 to $4.70 \mathrm{~dB}$. The trade-off schedules offer noise deltas of up to $\triangle E A O A S P L=-5.36 \mathrm{~dB}$ accompanied by environmental deltas of up to $\Delta N O_{x}^{\text {rate }}=-9.91 \%$. 


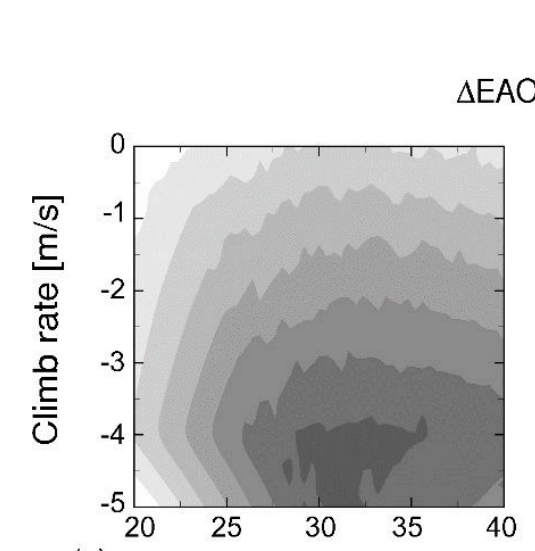

(a)

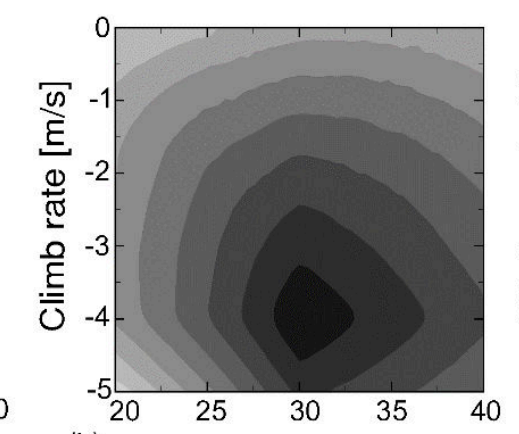

(b)

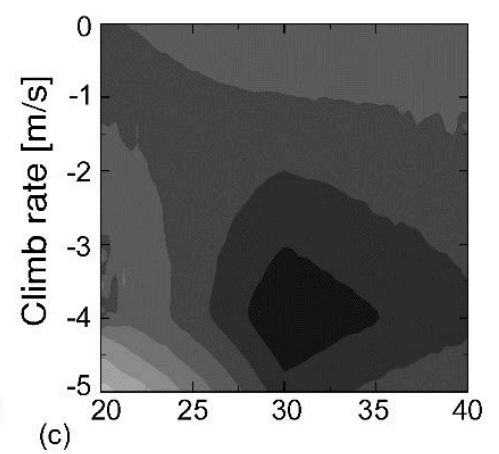

(c)

$\Delta \mathrm{NO}_{\mathrm{x}}[\%]:-\begin{array}{llllll}-20 & -12 & -4 & 4 & 12 & 20\end{array}$

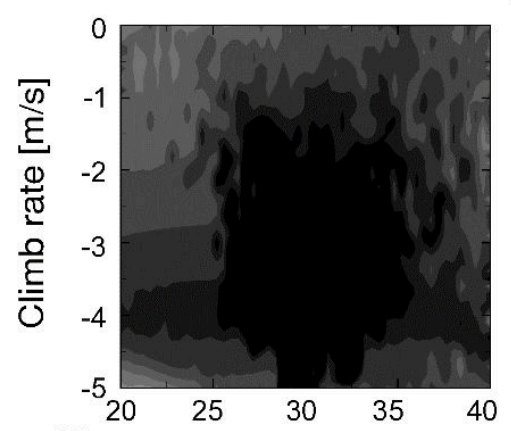

(d)

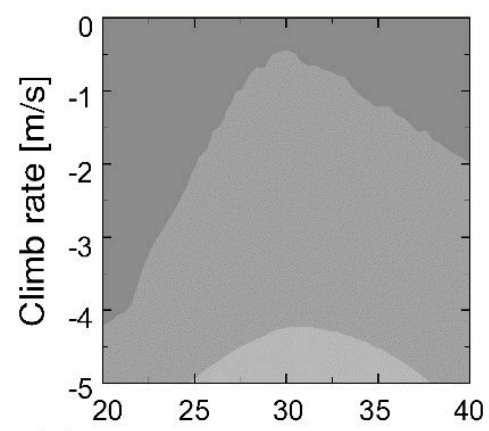

(e)

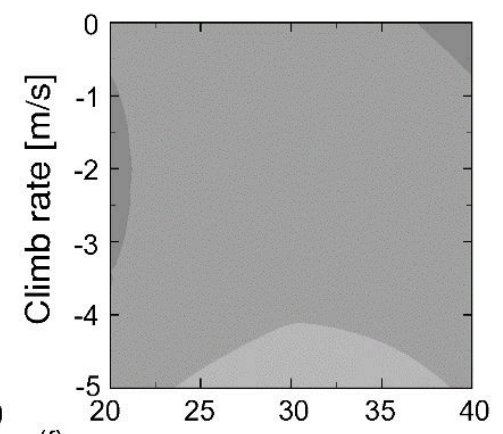

(f)

Fig. 10. EAOASPL and $N O_{x}$ benefits for $A U M=2000 \mathrm{~kg}:$ Noise benefits through optimizing for (a) EAOASPL, (b) best trade-off, (c) $N O_{x} ; N O_{x}$ benefits through optimizing for (d) EAOASPL, (e) best trade-off, (f) $N O_{x}$.

Figure 11 (a)-(f) presents the $\Omega^{O p t}$ and $\theta_{t w}^{O p t}$ schedules targeting at minimum noise, best tradeoff and minimum $N O_{x}$ emissions, respectively, for $A U M=2400 \mathrm{~kg}$. Generally, higher $\Omega^{O p t}$ and $\theta_{t w}^{O p t}$ is required compared to the $A U M=1600 \mathrm{~kg}$ and $A U M=2000 \mathrm{~kg}$ cases, with $\Omega^{O p t}$ reaching values up to $97.03 \%$ to minimize $N O_{x}^{\text {rate }}$ in low-speed steep descent. For noise minimization, $\theta_{t w}^{O p t}$ greater than $14 \mathrm{deg}$ is necessary to mitigate $E A O A S P L$ in level-flight conditions. Under these conditions, the lateral tip regions of the rotor are dominated by strong vortex roll-up. Increased $\theta_{t w}$ leads to an inboard shift of tip aerodynamic loading, yielding a 

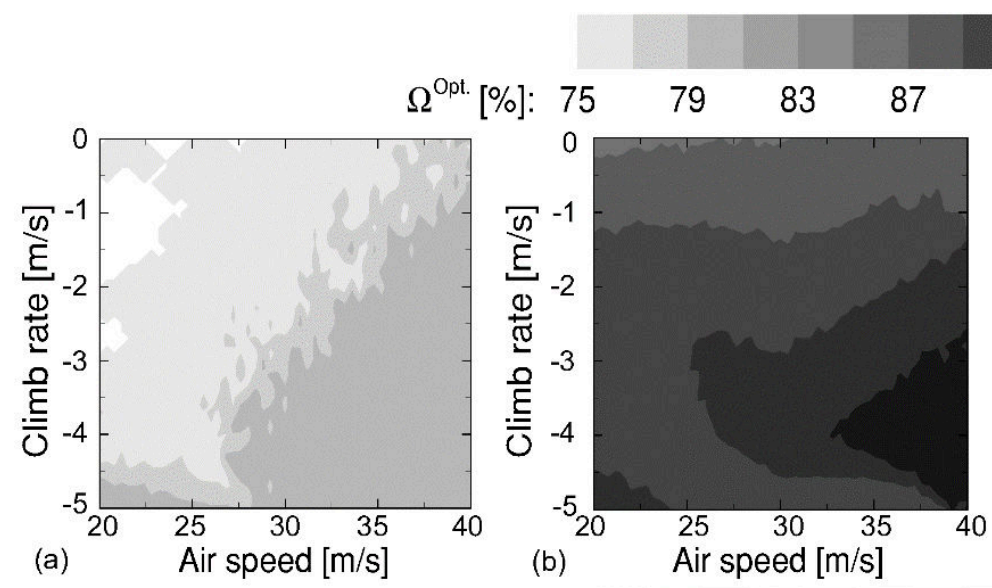

(b)

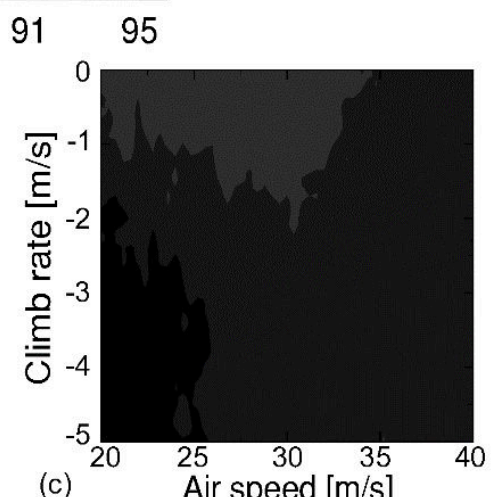

(c) $\quad$ Air speed $[\mathrm{m} / \mathrm{s}]$

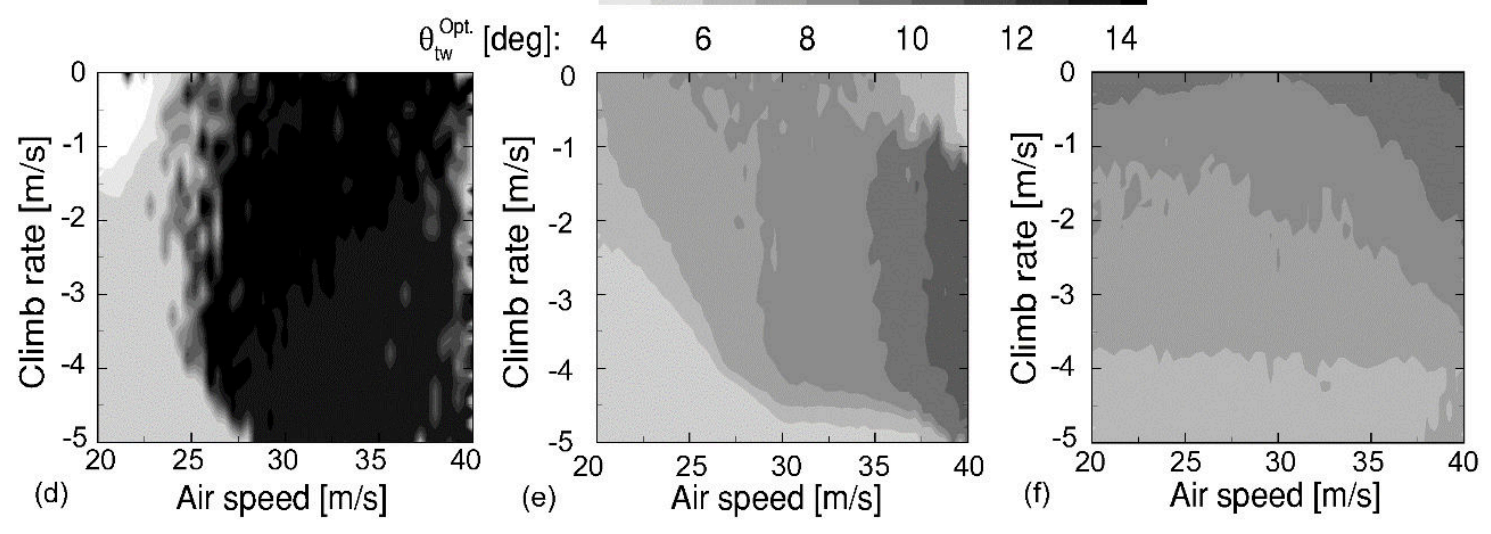

Fig. 11. Optimized rotor VRS and ABT schedules for $A U M=2400 \mathrm{~kg}$ : Optimal rotor speed for (a) EAOASPL, (b) best trade-off, (c) $N O_{x}$; Optimal blade twist angle for (d) EAOASPL , (e) best trade-off, (f) $N O_{x}$.

reduction of tip vortex strength and consequently loading noise generated from vortex roll-up. It can be concluded that the trade-off between noise and $N O_{x}$ is governed by $A U M$ : for high $A U M$ cases, insignificant $N O_{x}$ benefits are attained by reducing $\Omega$. Under these high-loaded conditions, the higher the reduction in $\Omega$, the higher the increase in rotor power due to the aerodynamic performance penalties imposed by the excessively increased blade loading to sustain trim. However, there are still noise benefits at the expense of higher $N O_{x}$ emissions. 


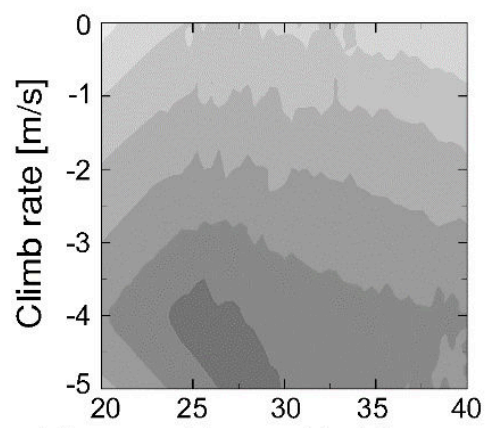

(a)
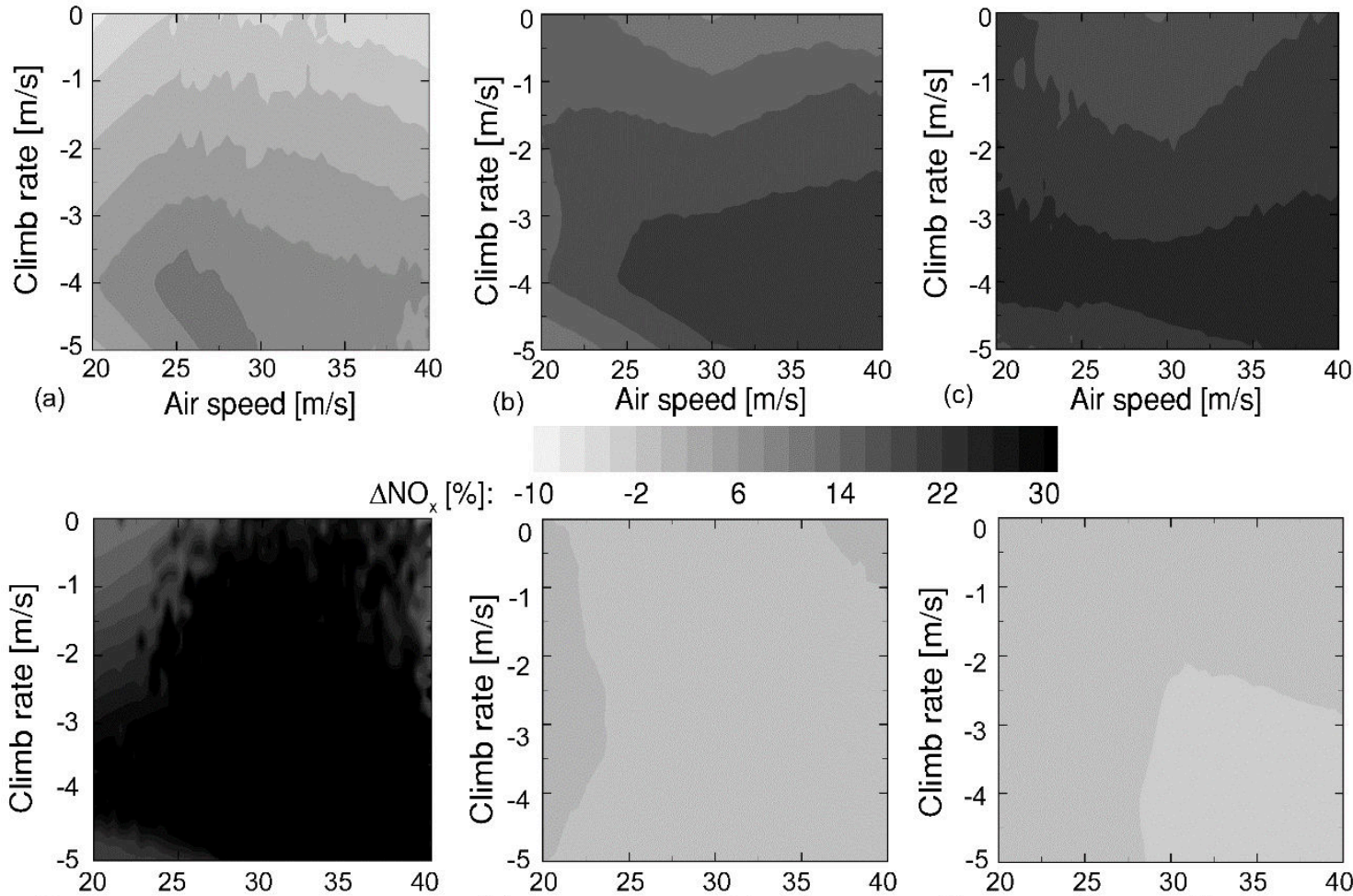

(d)

Air speed $[\mathrm{m} / \mathrm{s}]$

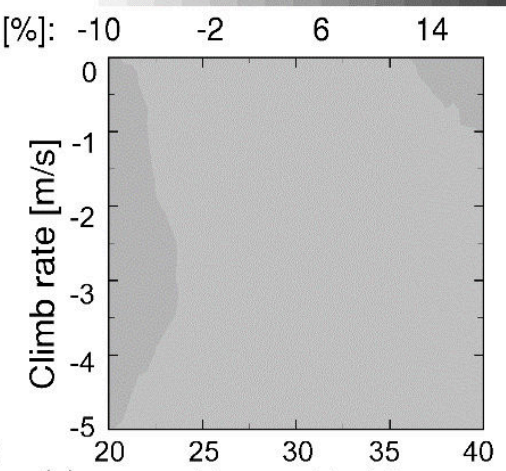

(e) Air speed $[\mathrm{m} / \mathrm{s}]$

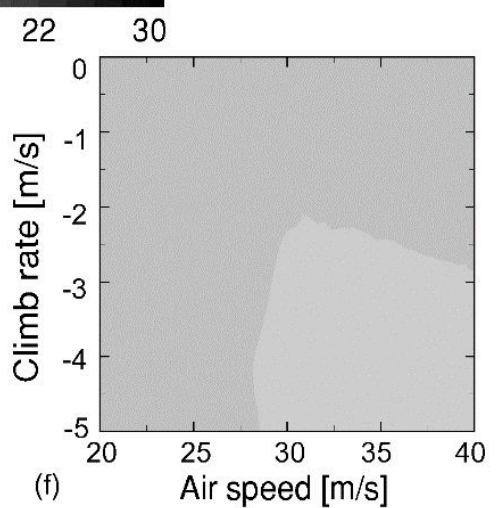

Fig. 12. EAOASPL and $N O_{x}$ benefits for $A U M=2400 \mathrm{~kg}$ : Noise benefits through optimizing for (a) EAOASPL, (b) best trade-off, (c) $N O_{x} ; N O_{x}$ benefits through optimizing for (a) EAOASPL, (b) best trade-off, (c) $N O_{x}$.

Figure 12 (a)-(f) reflects the obtained EAOASPL and $N O_{x}^{\text {rate }}$ reductions attainable by the application of the proposed schedules for $A U M=2400 \mathrm{~kg}$. In the trade-off case, it is observed that the acquired improvements are the smallest, compared to the $A U M=1600 \mathrm{~kg}$ and $A U M=2000 \mathrm{~kg}$ cases. Specifically, $\triangle E A O A S P L$ is ranging between -1.10 to $-3.08 \mathrm{~dB}$, whilst the highest attainable $N O_{x}$ benefit for the trade-off case is $\Delta N O_{x}^{\text {rate }}=-1.90 \%$, with negligible reductions or even slight increases of up to $0.79 \%$ at the greatest part of the descent flight envelope. 


\section{Conclusions}

This work quantified the potential improvement on environmental and acoustic impact stemming from the optimum implementation of variable rotor speed and active blade twist schedules for helicopters in descending flight. An integrated framework was deployed comprising models for helicopter aeromechanics, rotor aeroacoustics, engine performance and gaseous emissions. A multi-disciplinary optimization approach was presented, based on surrogate modeling and global, gradient-free optimization. The overall methodology was utilized to devise optimal schedules of combined variable rotor speed and active blade twist, applied on a representative twin-engine light helicopter, across a wide range of descent operating conditions. Three types of optimal schedules have been presented: $N O_{x}$-optimized, noise-optimized and the best trade-off between $N O_{x}$ and noise.

The importance of multi-disciplinary optimization has been demonstrated for an intermediate mass case: the noise-optimized schedules offered reductions up to $6.62 \mathrm{~dB}$, albeit at a cost of $60 \%$ increase in $N O_{x}$. The $N O_{x}$-optimized schedules provided $10.29 \%$ less $N O_{x}$ emissions, although noise reductions ranged between 1.07 to $4.70 \mathrm{~dB}$. The trade-off schedules achieved noise benefits of up to $5.36 \mathrm{~dB}$, simultaneously with $N O_{x}$ improvements of up to $9.91 \%$.

It has also been shown that the effectiveness of variable rotor speed and active blade twist is strongly dependent on rotorcraft all-up mass, as well as flight speed and climb rate. In low all-upmass conditions, the highest noise benefits have been accrued at steep descent, due to the effectiveness of active blade twist in counteracting the increase in angle of attack occurring by the high free-stream vertical velocity component. At flight path angles where blade-vortex interactions are more pronounced it has been shown that the concept cannot entirely mitigate the interactions, but still noise benefits of the order of $3 \mathrm{~dB}$ are attainable. The highest $N O_{x}$ reductions have been observed in steep descent, and specifically close to the speed of maximum endurance, where power requirements are low. Overall, in low all-up mass conditions, noise 
reductions between 2.31 and $8.30 \mathrm{~dB}$ have been calculated, along with concurrent $N O_{x}$ reductions of $6.42 \%$ to $21.32 \%$. Finally, in high all-up-mass conditions, the schedules that achieve the best trade-off between the conflicting objectives offered noise benefits in the range of 1.10 to $3.08 \mathrm{~dB}$ combined with $N O_{x}$ improvements of up to $1.90 \%$. Concluding, the developed strategy constitutes an enabling methodology for the holistic and multi-disciplinary assessment of the environmental and acoustic impact of morphing helicopter rotor configurations.

\section{Conflict of interest statement}

None declared.

\section{References}

[1] Federal Aviation Administration (2019) FAA Aerospace Forecast.

[2] Yunos, S., Ghafir, M. F. A. and Wahab, A. A. (2017) 'Aircraft LTO emissions regulations and implementations at European airports', AIP Conference Proceedings, 1831(1).

[3] ICAO (2008) 'Guidance on the Balanced Approach to Aircraft Noise Management', International Civil Aviation Organization, 9829.

[4] Misté, G. A., Benini, E., Garavello, A. and Gonzalez-Alcoy, M. (2015) 'A methodology for determining the optimal rotational speed of a variable RPM main rotor/turboshaft engine system', Journal of the American Helicopter Society, 60(3), pp. 1-11.

[5] Garavello, A. and Benini, E. (2012) 'Preliminary Study on a Wide-Speed-Range Helicopter Rotor / Turboshaft System', Journal of Aircraft, 49(4).

[6] Steiner, J. H. (2008) An Investigation of Performance Benefits and Trim Requirements of a 
Variable Speed Helicopter Rotor. MSc Dissertation, Pennsylvania State University.

[7] Bowen-Davies, G. M. and Chopra, I. (2015) 'Aeromechanics of a slowed rotor', Journal of the American Helicopter Society, 60(3), pp. 1-13.

[8] Bir, G., Chopra, I. and Nguyen, K. (1990) ‘Development of UMARC (University of Maryland Advanced Rotorcraft Code)'.

[9] Mistry, M. and Gandhi, F. (2014) 'Helicopter performance improvement with variable rotor radius and RPM', Journal of the American Helicopter Society, 59(4), pp. 17-35.

[10] Mueller, A. W., Childress, O. S. and Hardesty, M. (1987) Helicopter Main-Rotor Speed Effects on Far-Field Acoustic Levels.

[11] Pagano, A., Ameduri, S., Cokonaj, V., Prachar, A., Zachariadis, Z. and Drikakis, D. (2011) 'Helicopter blade morphing strategies aimed at mitigating environmental impact', Journal of Theoretical and Applied Mechanics, 49(4), pp. 1233-1259.

[12] Yung, H. Y., Gmelin, B., Splettstoesser, W., Philippe, J. J., Prieur, J. and Brooks, T. F. (1997) 'Reduction of helicopter blade-vortex interaction noise by active rotor control technology', Progress in Aerospace Sciences, 33(9-10), pp. 647-687.

[13] Jacklin, S. A., Blaas, A., Teves, D., Kube, R. and Warmbrodt, W. (1995) 'Reduction of helicopter BVI noise, vibration, and power consumption through individual blade control', in 51st American Helicopter Society Forum.

[14] Kube, R., Splettstoesser, W. R., Wagner, W., Seelhorst, U., Yu, Y. H., Tung, C., Beaumier, P., Prieur, J., Rahier, G., Spiegel, P., Boutier, A., Brooks, T. F., Burley, C. L., Boyd, D. D., Mercker, E. and Pengel, K. (1998) 'HHC aeroacoustic rotor tests in the German-Dutch wind tunnel: Improving physical understanding and prediction codes', Aerospace science and technology, 2(3).

[15] Wilbur, M. L., Mirick, P. H., Yeager, W. T., Langston, C. W., Cesnik, C. E. and Shin, S. 
(2002) 'Vibratory loads reduction testing of the NASA/Army/MIT active twist rotor', Journal of the American Helicopter Society, 47(2), pp. 123-133.

[16] Brooks, T. F. and Booth, E. R. (1993) 'The Effects of Higher Harmonic Control on BladeVortex Interaction Noise and Vibration', Journal of the American Helicopter Society, 38(3), pp. $45-55$.

[17] Bebesel, M., Roth, D. and Pongratz, R. (2002) 'Reduction of BVI noise on ground-in-flight evaluation of closed-loop controller', in 28th European Rotorcraft Forum.

[18] Booth, E. R. and Wilbur, M. L. (2004) 'Acoustic Aspects of Active- Twist Rotor Control', Journal of the American Helicopter Society, 49(1), pp. 3-10.

[19] Han, D., Pastrikakis, V. and Barakos, G. N. (2016) 'Helicopter performance improvement by variable rotor speed and variable blade twist', Aerospace Science and Technology. Elsevier Masson SAS, 54, pp. 164-173.

[20] Goulos, I. and Bonesso, M. (2019) 'Variable rotor speed and active blade twist for civil rotorcraft: Optimum scheduling, mission analysis, and environmental impact', Aerospace Science and Technology. Elsevier Masson SAS, 1, pp. 1-13.

[21] Goulos, I., Pachidis, V. and Pilidis, P. (2014) 'Lagrangian Formulation for the Rapid Estimation of Helicopter Rotor Blade Vibration Characteristics', The Aeronautical Journal, 118(1206), pp. 861-901.

[22] Goulos, I., Pachidis, V. and Pilidis, P. (2014) 'Helicopter Rotor Blade Flexibility Simulation for Aeroelasticity and Flight Dynamics Applications', Journal of the American Helicopter Society, 59(4), pp. 1-18.

[23] Goulos, I. (2016) ‘An Improved Analytical Approach for Modeling the Effect of Rotor Wake Curvature Using Finite-State Induced Flow Models’, American Helicopter Society, 61(3).

[24] Goulos, I., Giannakakis, P., Pachidis, V. and Pilidis, P. (2013) 'Mission Performance 
Simulation of Integrated Helicopter-Engine Systems Using an Aeroelastic Rotor Model', Journal of Engineering for Gas Turbines and Power, 135(9).

[25] Pardo, A. C., Goulos, I. and Pachidis, V. (2016) 'Modeling and analysis of coupled flap-lagtorsion vibration characteristics helicopter rotor blades', Proceedings of the Institution of Mechanical Engineers, Part G: Journal of Aerospace Engineering, 230(10), pp. 1804-1823.

[26] Leishman, J. G. and Beddoes, T. S. (1989) 'A Semi-Empirical Model for Dynamic Stall', Journal of the Americal Helicopter Society, 34(3), pp. 3-17.

[27] Peters, D. A., Boyd, D. D. and He, C. J. (1989) 'Finite-State Induced-Flow Model for Rotors in Hover and Forward Flight', Journal of the American Helicopter Society, 34(4), pp. 5-17.

[28] MacMillan, W. L. (1974) Development of a modular-type computer program for the calculation of gas turbine off-design performance. PhD Dissertation, Cranfield University.

[29] Celis, C., Moss, B. and Pilidis, P. (2009) 'Emissions Modeling for the Optimization of Greener Aircraft Operations', in Proceedings of ASME Turbo Expo 2009. Orlando, Florida, USA, pp. 167-178.

[30] Goulos, I., Ali, F., Tzanidakis, K., Pachidis, V. and D'Ippolito, R. (2015) 'A Multidisciplinary Approach for the Comprehensive Assessment of Integrated RotorcraftPowerplant Systems at Mission Level', Journal of Engineering for Gas Turbines and Power, 119(4215), pp. 891-914.

[31] Ortiz-Carretero, J., Castillo Pardo, A., Goulos, I. and Pachidis, V. (2017) 'Impact of Adverse Environmental Conditions on Rotorcraft Operational Performance and Pollutant Emissions', Journal of Engineering for Gas Turbines and Power, 140(2).

[32] Castillo Pardo, A. (2017) Aeroelastic Simulation of Rotorcraft Propulsion Systems. PhD Dissertation, Cranfield University.

[33] Bagai, A. and Leishman, J. G. (1995) 'Rotor Free-Wake Modeling using a Pseudo-Implicit 
Technique - Including Comparisons with Experimental Data', Journal of the American Helicopter Society, 40(3), pp. 29-41.

[34] van der Wall, B. G., Bauknecht, A., Jung, S. N. and You, Y. H. (2014) 'Semi-Empirical Physics-Based Modeling of Fuselage-Rotor and Fuselage-Wake Interferences for Comprehensive Codes', in 70th American Helicopter Society Annual Forum. Montreal, Quebec, Canada.

[35] van der Wall, B. G., Lim, J. W., Smith, M. J., Jung, S. N., Bailly, J., Baeder, J. D. and Douglas Boyd, D. (2014) 'The HART II international workshop: An assessment of the state-of-the-art in comprehensive code prediction', CEAS Aeronautical Journal, 4(3), pp. 223-252.

[36] Vouros, S., Goulos, I. and Pachidis, V. (2019) 'Integrated methodology for the prediction of helicopter rotor noise at mission level', Aerospace Science and Technology, 89, pp. 136-149.

[37] Vouros, S. (2019) Aeroacoustic Simulation of Rotorcraft Propulsion Systems. PhD Dissertation, Cranfield University.

[38] Farassat, F. (2007) Derivation of Formulations 1 and 1 A of Farassat. NASA/TM-214853.

[39] Farassat, F. and Succi, G. P. (1982) 'The Prediction of Helicopter Rotor Discrete Frequency Noise', in 38th American Helicopter Society Annual Forum. Washington, DC.

[40] Ffowcs Williams, J. E. and Hawkings, D. L. (1969) 'Sound Generation by Turbulence and Surfaces in Arbitrary Motion', Philosophical Transactions of the Royal Society of London, Series A, Mathematical and Physical Sciences, 264(1151), pp. 321-342.

[41] Lopes, L. V. (2017) 'Compact Assumption Applied to the Monopole Term of Farassat' s Formulations', Journal of Aircraft, 54(5), pp. 1649-1663.

[42] Brentner, K. S., Burley, C. L. and Marcolini, M. A. (1994) 'Sensitivity of Acoustic Predictions to Variation of Input Parameters', Journal of the American Helicopter Society, 39(3), pp. $43-52$. 
[43] Pegg, R. (1979) A Summary and Evaluation of Semi-Empirical Methods for the Prediction of Helicopter Rotor Noise. NASA TM, vol. 80200.

[44] Tsuchiya, T., Ikaida, H., Ishii, H., Gomi, H. and Okuno, Y. (2011) 'Real-Time Trajectory Optimization for Noise Abatement of Helicopter Landings', Journal of Mechanical Systems for Transportation and Logistics, 4(2), pp. 95-110.

[45] Ikaida, H., Tsuchiya, T., Ishii, H., Gom, H. and Okuno, Y. (2010) 'Numerical Simulation of Real-Time Trajectory Optimization for Helicopter Noise Abatement', Journal of Mechanical Systems for Transportation and Logistics, 3(2), pp. 415-430.

[46] Bayraktar, H. and Turalioglu, S. F. (2005) ‘A Kriging-based approach for locating a sampling site - in the assessment of air quality', Stochastic Environmental Research and Risk Assessment, 19(4), pp. 301-305.

[47] Kohavi, R. (1995) ‘A Study of Cross-Validation and Bootstrap for Accuracy Estimation and Model Selection', in International Joint Conference on Artificial Intelligence.

[48] Deb, K., Pratap, A., Agarwal, S. and Meyarivan, T. (2002) 'A Fast and Elitist Multiobjective Genetic Algorithm : NSGA-II', IEEE Transactions of Evolutionary Computation, 6(2), pp. 182197.

[49] Mistry, M. and Gandhi, F. (2014) 'Helicopter Performance Improvement with Variable Rotor Radius and RPM', Journal of the American Helicopter Society, 59(4), pp. 17-35.

[50] Walsh, P. and Fletcher, P. (2004) Gas Turbine Performance. 2nd edn. John Wiley \& Sons.

[51] Bowen-Davies, G. M. and Chopra, I. (2015) 'Aeromechanics of a Slowed Rotor', Journal of the American Helicopter Society, 60(3), pp. 1-13.

[52] Leishman, J. G. (2006) Principles of Helicopter Aerodynamics. 2nd edn. Edited by W. Shyy and M. J. Rycroft. Cambridge University Press. 
[53] Gu, X., Sun, G., Li, G., Mao, L. and Li, Q. (2013) 'A comparative study on multiobjective reliable and robust optimization for crashworthiness design of vehicle structure', Structural and Multidisciplinary Optimization, 48(3), pp. 669-684. 


\section{Multi-disciplinary optimization of variable rotor speed and active blade twist} rotorcraft: Trade-off between noise and emissions

Polyzos, Nikolaos D.

Elsevier

Polyzos ND, Vouros S, Goulos I, Pachidis V. (2020) Multi-disciplinary optimization of variable rotor speed and active blade twist rotorcraft: Trade-off between noise and emissions. Aerospace Science and Technology, Volume 107, December 2020, Article number 106356 https://doi.org/10.1016/j.ast.2020.106356

Downloaded from Cranfield Library Services E-Repository 